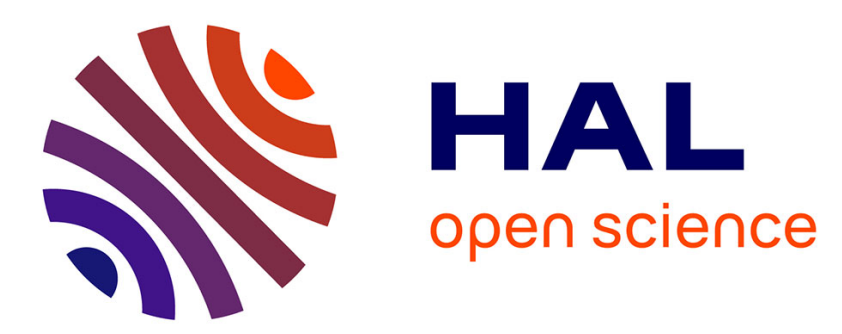

\title{
A probabilistic model for bounded elasticity tensor random fields with application to polycrystalline microstructures
}

\author{
Johann Guilleminot, A. Noshadravan, Christian Soize, R. Ghanem
}

\section{- To cite this version:}

Johann Guilleminot, A. Noshadravan, Christian Soize, R. Ghanem. A probabilistic model for bounded elasticity tensor random fields with application to polycrystalline microstructures. Computer Methods in Applied Mechanics and Engineering, 2011, 200 (17-20), pp.1637-1648. 10.1016/j.cma.2011.01.016 . hal-00684305

\section{HAL Id: hal-00684305 \\ https://hal.science/hal-00684305}

Submitted on 1 Apr 2012

HAL is a multi-disciplinary open access archive for the deposit and dissemination of scientific research documents, whether they are published or not. The documents may come from teaching and research institutions in France or abroad, or from public or private research centers.
L'archive ouverte pluridisciplinaire HAL, est destinée au dépôt et à la diffusion de documents scientifiques de niveau recherche, publiés ou non, émanant des établissements d'enseignement et de recherche français ou étrangers, des laboratoires publics ou privés. 


\title{
A probabilistic model for bounded elasticity tensor random fields with application to polycrystalline microstructures
}

\author{
J. Guilleminot*,a, A. Noshadravan ${ }^{\mathrm{b}}$, C. Soize ${ }^{\mathrm{a}}$, R. G. Ghanem ${ }^{\mathrm{b}}$ \\ ${ }^{a}$ Université Paris-Est, Laboratoire Modélisation et Simulation Multi Echelle, MSME \\ UMR8208 CNRS, 5 Bd Descartes, 77454 Marne la Vallée, France \\ ${ }^{b} 210$ KAP Hall, University of Southern California, Los Angeles, CA 90089, USA
}

\begin{abstract}
In this paper, we address the construction of a prior stochastic model for nonGaussian deterministically-bounded positive-definite matrix-valued random fields in the context of mesoscale modeling of heterogeneous elastic microstructures. We first introduce the micromechanical framework and recall, in particular, Huet's Partition Theorem. Based on the latter, we discuss the nature of hierarchical bounds and define, under some given assumptions, deterministic bounds for the apparent elasticity tensor. Having recourse to the Maximum Entropy Principle under the constraints defined by the available information, we then introduce two random matrix models. It is shown that an alternative formulation of the boundedness constraints further allows constructing a probabilistic model for deterministically-bounded positive-definite matrix-valued random fields. Such a construction is presented and relies on a class of random fields previously defined. We finally exemplify the overall methodology considering an experimental database obtained from EBSD measurements and provide a simple numerical application.
\end{abstract}

Key words: Micromechanics; Heterogeneous materials; Apparent elasticity tensor; Mesoscale modeling; Random field; Non-Gaussian.

\footnotetext{
${ }^{2}$ J. Guilleminot, A. Noshadravan, R. Ghanem and C. Soize, A probabilistic model for bounded elasticity tensor random fields with application to polycrystalline microstructures, Computer Methods in Applied Mechanics and Engineering, 200(17-20), 1637-1648 (2011).

${ }^{*}$ Corresponding author

Email address: johann.guilleminot@univ-paris-est.fr (J. Guilleminot)
} 


\section{Introduction}

The general area of stochastic multiscale modeling of complex multiphase random materials (such as some fiber reinforced composites or living tissues) has gained a continuing interest among the scientific community during the past two decades. Historically, most of the attempts were derived having recourse to the numerical simulations of random microstructures (relying on the experimental identification of some morphological characteristics, or assuming such properties; see [38]), coupled with a (Stochastic) finite elements analysis [5] (see for instance [12] [37]) and a statistical study performed on any quantity of interest (local strain or stress random fields, etc.).

Besides these quantitative studies, it is desirable to develop mathematical representations that, while ensuring a set of fundamental algebraic and probabilistic properties on the modeled quantities (as well as on the stochastic response of the system, should the modeling be used in the formulation of a stochastic boundary value problem for instance), catch as many physical and mechanical information as possible. Such models turn out to be necessary when performing an experimental identification solving an inverse problem or introducing the underlying subscale randomness into coarse scale computational models for instance. In this context, the Maximum Entropy (MaxEnt) principle has been shown to be an efficient method allowing the explicit construction of prior probability distributions while considering the available information only (see Section 2.2).

Based on the so-called nonparametric approach for uncertainties derived in [31] [32] (making use of the MaxEnt principle), a class for positive-definite matrix-valued random fields (extending the class previously constructed in [33]), corresponding to the prior probability model of an elastic anisotropic microstructure, was proposed in [34]. Invoking the energy-based boundedness constraints on the random elasticity tensor established by Huet (see [10]), a random matrix approach has been derived later on in [2] (see Section

2.3.1). Noticing that the two above approaches induce anisotropic statistical fluctuations which may not be encountered in practice (and in particular, in geophysical applications), Ta and his coworkers proposed a refinement of the probabilistic model derived by Soize by introducing a new parameter controlling the anisotropy index apart from the level of fluctuations [36]. Such developments are basically in the class of the generalized probabilistic approach of uncertainties corresponding to a coupling between the parametric probabilistic approach and the nonparametric one; see [35]. An alternative and more general derivation (in the sense that the distance to a given symmetry class is not restricted to the isotropic case), based on a 
particular characterization of the material symmetry groups and consisting in prescribing the variances of some stochastic eigenvalues of the random elasticity matrix, has been finally studied in [6]. Such an approach allows modeling random elasticity tensors whose mean distance to a given material symmetry class is specified. Note that the Maximum Entropy principle has also been used, within a parametric framework, for simulating random microstructures in accordance with some morphological features [25].

The present research is devoted to the construction of a prior probabilistic model for deterministically-bounded positive-definite matrix-valued random fields. Here, the term 'nonparametric' makes reference to the fact that none of the random (morphological) parameters that could be used to describe the random microstructure (e.g. the local volume fraction, the shapes of the heterogeneities, etc.) is explicitly considered and modeled. The induced probability measure is specified by the MaxEnt procedure and constraints synthesized from experimental data. The paper generalizes results previously obtained on bounded random elasticity matrices (see [2]) so as to facilitate the development of an associated random field model.

This paper is organized as follows. Section 2 deals with the construction of the prior probabilistic model for positive-definite random matrices that are bounded, in a deterministic sense, from above and below. In particular, we introduce and discuss, within a micromechanical framework, the boundedness constraints that are considered in the formulation of the MaxEnt principle. We then present, in Section 3, the probabilistic model for nonGaussian deterministically-bounded positive-definite matrix-valued random fields. An application, based on a set of experimental data and illustrating the overall methodology, is finally provided in Section 4.

\section{Probabilistic models for deterministically bounded symmetric positive-definite real matrices}

Let $\mathbb{M}_{n}^{S}(\mathbb{R})$ and $\mathbb{M}_{n}^{+}(\mathbb{R}) \subset \mathbb{M}_{n}^{S}(\mathbb{R})$ be the set of all the $(n \times n)$ real symmetric and symmetric positive-definite matrices respectively, with $n \leq 6$. A generic point of the Euclidean space $\mathbb{R}^{3}$ is denoted by $\mathbf{x}=\left(x_{1}, x_{2}, x_{3}\right)$. Notation $\mathbf{x}^{(i)}$ refers to the i-th point defined with respect to any indexation system. In what follows, single and double brackets denote matrix-valued and tensor-valued variables, respectively.

In this section, we address the construction of a prior probabilistic model for a suitable $\mathbb{M}_{n}^{+}(\mathbb{R})$-valued random matrix representation $[\mathbf{L}]$ of the fourthorder random elasticity tensor $[[\mathbf{L}]]$, under the additional constraint that each realization of the matrix-valued random variable $[\mathbf{L}]$ is bounded (in 
the usual quadratic sense) from above and below. In order to make the paper self-contained, the definition of such bounds is first introduced and thoroughly discussed within the classical framework of micromechanics in Section 2.1. In particular, the stationarity and ergodicity properties are recalled in the context of stochastic homogenization. Following the pioneering work by Soize (see [31] [32]), the construction of the probabilistic models is based on the use of the Maximum Entropy principle and is recalled in Section 2.2. Two probabilistic models are then presented. The first one was recently proposed in the literature and is briefly reviewed in Section 2.3.1. Making use of an alternative formulation of the boundedness constraint and having recourse to a change of variable, we subsequently show that the probabilistic model defined in [31] [32], in which the probability distribution is supported over $\mathbb{M}_{n}^{+}(\mathbb{R})$, can be used as well. In particular, such a derivation turns out to be very useful in order to extend the modeling to the random field case, as will be seen in Section 3 .

\subsection{Micromechanics-based constraints for the elasticity tensor of random heterogeneous media}

\subsubsection{Remarks about the stationarity and ergodicity properties in the context of stochastic homogenization}

Let $\Omega \subset \mathbb{R}^{3}$ be a domain with boundary $\partial \Omega$, occupied by a heterogeneous random medium. Let $\mathbf{x} \mapsto \mathbf{S}(\mathbf{x})$ be a random field defined on a probability space $(\Theta, \mathcal{T}, \mathcal{P})$, indexed by $\mathbb{R}^{3}$ and with values in a subset of a vector space $\mathbb{H}_{\mathbf{S}}$, describing the local morphology and/or the elastic properties of the microstructure. For all $\theta$ in $\Theta$, one has $\mathbf{S}(\mathbf{x}, \theta)$ in $\mathbb{H}_{\mathbf{S}}$ and $\mathbf{x} \mapsto \mathbf{S}(\mathbf{x}, \theta)$ is a trajectory of $\mathbf{S}$ which is assumed to belong to a set of functions $\mathcal{H}_{\mathbf{S}}$. Consequently, the random field $\mathbf{S}$ is a family of random variables defined on $(\Theta, \mathcal{T}, \mathcal{P})$ with values in $\mathcal{H}_{\mathbf{S}}$. Let $g$ be a (measurable) functional mapping from $\mathcal{H}_{\mathbf{S}}$ in $\mathcal{H}_{g}$, where, for all $\mathbf{s}$ in $\mathcal{H}_{\mathbf{S}}, \mathcal{H}_{g}$ is a set of functions $\{\mathbf{x} \mapsto\{g(\mathbf{s})\}(\mathbf{x})\}$ defined on $\Omega$ with values in $\mathbb{H}_{g}$. Let $<\{g(\mathbf{S})\}>$ be the random variable, corresponding to the modeling of any homogenized quantity of interest (such as the effective stress or strain tensor), defined as:

$$
<\{g(\mathbf{S})\}>_{\Omega}=\frac{1}{|\Omega|} \int_{\Omega}\{g(\mathbf{S})\}(\mathbf{x}) d \mathbf{x} .
$$

Therefore, the effective property $<\{g(\mathbf{S})\}>_{\Omega}$ is a $\mathbb{H}_{g}$-valued random variable whose probability distribution $P_{<\{g(\mathbf{S})\}>_{\Omega}}$ is completely defined by the probability law of random field $x \mapsto \mathbf{S}(\mathbf{x})$ and by mapping $g$. We assume that transformation $g$ and random field $\mathbf{S}$ are such that $\mathbf{x} \mapsto\{g(\mathbf{S})\}(\mathbf{x})$ is 
a homogeneous random field indexed by $\mathbb{R}^{3}$ (which means that its system of marginal probability distributions is invariant under translation in $\mathbb{R}^{3}$ ). In addition, if the homogeneous random field $\mathbf{x} \mapsto\{g(\mathbf{S})\}(\mathbf{x})$ is shown (or assumed) to be ergodic in the mean-square sense (note that the mean-square convergence can be replaced by any other mode of convergence) with respect to the mean function $m_{\{g(\mathbf{S})\}}=\mathrm{E}\{\{g(\mathbf{S})\}(\mathbf{x})\}$ (independent of $\mathbf{x}$ ) of $\{g(\mathbf{S})\}$, we then have:

$$
\lim _{\Omega \rightarrow \mathbb{R}^{3}} \mathrm{E}\left\{\left\|<\{g(\mathbf{S})\}>_{\Omega}-m_{\{g(\mathbf{S})\}}\right\|^{2}\right\}=0,
$$

in which $\|\cdot\|$ is a suitable norm in $\mathbb{H}_{g}$. Consequently, for all $\varsigma>0$, there exists a domain $\Omega_{\varsigma}$ such that:

$$
\mathrm{E}\left\{\left\|<\{g(\mathbf{S})\}>_{\Omega_{\varsigma}}-m_{\{g(\mathbf{S})\}}\right\|^{2}\right\} \leq \varsigma .
$$

If Eq. (3) holds for a given and sufficiently small $\varsigma$, then the random variable $<\{g(\mathbf{S})\}>_{\Omega_{\varsigma}}$ exhibits a negligible level of statistical fluctuations and consequently, $\Omega_{\varsigma}$ is called the Representative Volume Element (RVE). The effective mechanical property, associated with this RVE, is almost deterministic. If the size of domain $\Omega_{\varsigma}$ is much smaller than the characteristic length of the macroscale, one can state the separation between the microscale (RVE) and the macroscale.

From Eq. (1), it is readily seen that the construction of the probability law of random field $\{g(\mathbf{S})\}$ allows for the calculation of the probability distribution of random variable $<\{g(\mathbf{S})\}>_{\Omega}$. Consequently, a probabilistic analysis of the RVE size can be carried out as soon as the probability law of random field $\{g(\mathbf{S})\}$ is known. It should also be noted that the meanergodicity assumption, allowing one to substitute spatial averaging (over the RVE) for statistical averaging, is absolutely required for performing any homogenization procedure if the above probability distribution is unknown.

In this paper, we make use of these derivations and consider the mesoscale modeling of a stationary random microstructure. More specifically, $\mathbf{x} \mapsto$ $\{g(\mathbf{S})\}(\mathbf{x})$ models the mesoscopic elasticity tensor random field and $\mathbf{S}$ describes the morphology and/or the properties of the underlying microstructure at microscale. For this type of analysis, random field $\mathbf{S}$ is not explicitly described but the construction of the probability law of random field $\{g(\mathbf{S})\}$ is directly addressed using neither $g$ nor $\mathbf{S}$. In this context, the definition of the RVE size through a probabilistic convergence analysis on the apparent properties has been introduced in [34]. 


\subsubsection{Definition of the apparent elasticity tensors}

A fundamental property of the effective elasticity tensor is that it does not depend on the usually prescribed boundary conditions (that are, the kinematic and static uniform boundary conditions; see below). In this context, the theoretical calculation of either estimates or bounds on the overall properties of random heterogeneous materials gave rise to an extensive literature in micromechanics and homogenization theories (see [16] [19] and the references therein). Such a deterministic framework was proven to be suitable for modeling random medium for which the typical size of the constituents is very small compared to the dimensions defining the coarse scale (that is, the scale at which the engineering structural application is carried out, for instance).

However, there exists a few classes of materials, for example some concretes or (long) fiber-reinforced composites, for which the scale separation can not be stated. In this case, statistical fluctuations still remain at the macroscale and the experimental characterization is often carried out for bodies smaller than the RVE, called Statistical Volume Elements. Their associated mechanical properties are then called apparent properties and are, by definition, random. Thus, the apparent tensor depends, unlike the effective one, on both the realization of the random medium and on the applied boundary conditions. This fact partly explains the large amount of scattering encountered in experimental testing on such materials.

The relation between the effective and apparent tensors was first studied by Huet [10] (see also the probabilistic interpretation by Sab [24], as well as the general review provided in [21]). In this paper, we denote by $\left[L^{\mathrm{eff}}\right]$ (resp. $\left.\left[\mathbf{L}^{\text {app }}\right]\right)$ the matrix representation of the effective (resp. apparent) elasticity tensor $\left[\left[L^{\mathrm{eff}}\right]\right]$ (resp. $\left.\left[\left[\mathbf{L}^{\mathrm{app}}\right]\right]\right)$. It should further be pointed out that the matrix representation of the elasticity tensor must be chosen in a way that ensures the equivalence between quadratic forms written using a tensorial formalism and those expressed having recourse to a matrix representation. Such an equivalence can be obtained making use of the well-known VoigtMandel matrix representation for instance, and is assumed in the following. Thus, any inequality (see Sections 2.1.3 and 2.1.4) will be written in matrix form. Below, we briefly review the main and fundamental results derived in [10].

Following the notation introduced in the previous section, for any integrable function $\mathbf{x} \mapsto a(\mathbf{x})$ (defined from $\Omega$ into $\mathbb{H}_{a}$ ), we denote by $\langle a\rangle$ the volume average of $a$ over $\Omega$ (note that for notational convenience, de- 
pendance of $\langle a\rangle$ on $\Omega$ is now dropped), namely:

$$
<a>=\frac{1}{|\Omega|} \int_{\Omega} a(\mathbf{x}) d \mathbf{x} .
$$

For a domain $\Omega$ equal or larger than the RVE, the effective elasticity tensor $\left[\left[L^{\mathrm{eff}}\right]\right]$ can thus be defined as:

$$
<\sigma>=\left[\left[L^{\mathrm{eff}}\right]\right]:<\epsilon>
$$

where the symbol ":" denotes the classical twice contracted tensor product. For $\mathbf{x} \in \Omega$, the functions $\mathbf{x} \mapsto \sigma(\mathbf{x})$ and $\mathbf{x} \mapsto \epsilon(\mathbf{x})$ denote the local stress and strain fields, respectively.

We recall that the Hill condition (also known as the Hill's macrohomogeneity condition or Hill-Mandel condition) states the following equivalence $[9]:$

$$
<\sigma: \epsilon>=<\sigma>:<\epsilon>\text {. }
$$

We are now in a position to define two essential kinds of boundary conditions, as well as the associated apparent tensors. Unless otherwise stated, domain $\Omega$ is assumed to be smaller than the RVE.

In the first case, one assumes the prescription of a displacement field of the form:

$$
\mathbf{u}(\mathbf{x})=\epsilon_{0} \mathbf{x}, \quad \forall \mathbf{x} \in \partial \Omega,
$$

to the boundary of the domain $\Omega$. In Eq. (7), $\epsilon_{0}$ is a given symmetric second-order tensor whose components have magnitudes consistent with the retained framework of small deformations. Following the original terminology from Huet, we refer to this kind of boundary conditions as kinematic uniform boundary conditions (KUBC). Due to the linearity of the problem, one can write:

$$
<\sigma>=\left[\left[\mathbf{L}_{\epsilon}^{\mathrm{app}}\right]\right]:<\epsilon>=\left[\left[\mathbf{L}_{\epsilon}^{\mathrm{app}}\right]\right]: \epsilon_{0},
$$

in which the fourth-order tensor $\left[\left[\mathbf{L}_{\epsilon}^{\text {app }}\right]\right]$ denotes the so-called kinematic apparent modulus (or elasticity) tensor of $\Omega$ in KUBC. The kinematic apparent compliance tensor $\left[\left[\mathbf{M}_{\epsilon}^{\mathrm{app}}\right]\right]$ of body $\Omega$ in $\mathrm{KUBC}$ is readily obtained by inverting Eq. (8), so that:

$$
\left[\left[\mathbf{M}_{\epsilon}^{\mathrm{app}}\right]\right]=\left[\left[\mathbf{L}_{\epsilon}^{\mathrm{app}}\right]\right]^{-1} .
$$

In the second case, one considers that a traction vector field of the following form is applied:

$$
\mathbf{t}(\mathbf{x})=\sigma_{0} \mathbf{n}(\mathbf{x}), \quad \forall \mathbf{x} \in \partial \Omega
$$


where $\mathbf{n}(\mathbf{x})$ is the unit vector normal to boundary $\partial \Omega$ at point $\mathbf{x}$ and $\sigma_{0}$ is a given symmetric second-order tensor, the components of which have magnitudes compatible with the elastic domain of the material under consideration. Such boundary conditions are referred to as static uniform boundary conditions (in brief, SUBC) and allow the definition of the static apparent compliance tensor $\left[\left[\mathbf{M}_{\sigma}^{\mathrm{app}}\right]\right]$ of $\Omega$ in SUBC, such as:

$$
<\epsilon>=\left[\left[\mathbf{M}_{\sigma}^{\mathrm{app}}\right]\right]:<\sigma>=\left[\left[\mathbf{M}_{\sigma}^{\mathrm{app}}\right]\right]: \sigma_{0} .
$$

Finally, one readily defines the static apparent modulus tensor of domain $\Omega$ in SUBC as:

$$
\left[\left[\mathbf{L}_{\sigma}^{\mathrm{app}}\right]\right]=\left[\left[\mathbf{M}_{\sigma}^{\mathrm{app}}\right]\right]^{-1} \text {. }
$$

It can easily be shown that both KUBC and SUBC satisfy the Hill condition (6) (note that the demonstration does not require the stationarity and ergodicity assumptions to be satisfied), so that the above mechanical definitions of the apparent tensors coincide with their energetic counterpart (see [10]).

\subsubsection{Inequalities between effective and apparent tensors: the Partition The- orem}

Let us assume in this section that $\Omega$ is equal or larger than the RVE, and divide it into $n$ equally shaped subdomains $V_{i}, i=1, \ldots, n$. For each subdomain $V_{i}$, we denote by $\left[\mathbf{L}_{\epsilon}^{\mathrm{app}}\left(V_{i}\right)\right]$ and $\left[\mathbf{M}_{\sigma}^{\mathrm{app}}\left(V_{i}\right)\right]$ the kinematic apparent elasticity matrix and the static apparent compliance matrix of $V_{i}$ in KUBC and SUBC, respectively. It can then be shown that:

$$
\left[\widehat{\mathbf{L}}_{\sigma}^{\mathrm{app}}\right] \leq\left[L^{\mathrm{eff}}\right] \leq\left[\widehat{\mathbf{L}}_{\epsilon}^{\mathrm{app}}\right]
$$

where the matrices $\left[\widehat{\mathbf{L}}_{\sigma}^{\text {app }}\right]$ and $\left[\widehat{\mathbf{L}}_{\epsilon}^{\text {app }}\right]$, called the static and kinematic statistical apparent modulus matrices, are defined by:

$$
\begin{gathered}
{\left[\widehat{\mathbf{L}}_{\sigma}^{\mathrm{app}}\right]=\left(\frac{1}{n} \sum_{i=1}^{n}\left[\mathbf{M}_{\sigma}^{\mathrm{app}}\left(V_{i}\right)\right]\right)^{-1}} \\
{\left[\widehat{\mathbf{L}}_{\epsilon}^{\mathrm{app}}\right]=\frac{1}{n} \sum_{i=1}^{n}\left[\mathbf{L}_{\epsilon}^{\mathrm{app}}\left(V_{i}\right)\right] .}
\end{gathered}
$$

Eq. (13) is referred to as the Partition Theorem [10]. As all stochastic inequalities that will be defined throughout this section, Eq. (13) means that for all $\theta_{k}$ in $\Theta$ :

$$
\left[\widehat{L}_{\sigma}^{\mathrm{app}}\left(\theta_{k}\right)\right] \leq\left[L^{\mathrm{eff}}\right] \leq\left[\widehat{L}_{\epsilon}^{\mathrm{app}}\left(\theta_{k}\right)\right],
$$


in which $\left[\widehat{L}_{\sigma}^{\mathrm{app}}\left(\theta_{k}\right)\right]$ and $\left[\widehat{L}_{\epsilon}^{\mathrm{app}}\left(\theta_{k}\right)\right]$ are the realizations of the static and kinematic statistical apparent elasticity matrices, associated with the realization $\theta_{k}$ of the random medium occupying domain $\Omega$. The non-strict inequality (13) ensures that the effective properties are recovered for $n=1$ (since KUBC and SUBC are then applied on a body equal or larger than the RVE, the properties of which are independent of the kind of boundary conditions). For the general case however (that is, for $n>1$ and subdomains $V_{i}$ smaller than the RVE), Eq. (13) is assumed to hold in the strict sense, so that:

$$
\left[\widehat{\mathbf{L}}_{\sigma}^{\mathrm{app}}\right]<\left[L^{\mathrm{eff}}\right]<\left[\widehat{\mathbf{L}}_{\epsilon}^{\mathrm{app}}\right] .
$$

For any real symmetric matrices $[A]$ and $[\mathrm{B}]$, inequality $[A]>[B]$ (resp. $[A] \geq[B]$ ) means that the matrix $[A]-[B]$ is positive-definite (resp. positivesemidefinite). Such a positive semidefinite ordering is usually referred to as Loewner ordering. It is worth noticing that no general conclusion about the (deterministic or random) nature of the bounds can be drawn from Eqs. (14-15), except for the limiting cases $n=1$ and $n \rightarrow+\infty$. In the first case, one recovers the deterministic effective elasticity matrix (as discussed above). For $n \rightarrow+\infty$ (in the sense that $n$ is "sufficiently" large, so that the elasticity or compliance matrix is homogeneous over each subdomain $V_{i}$ ), the bounds are readily seen to coincide with the classical, deterministic, Reuss (lower) and Voigt (upper) bounds. Finally, it should be noted that similar results hold for the effective and apparent compliance matrices (see $[10])$.

\subsubsection{Inequalities between apparent tensors at various mesoscales}

Let us now consider the case where $\Omega$ is smaller than the RVE. Consequently, we can define the corresponding static and kinematic statistical apparent elasticity matrices, denoted respectively by $\left[\mathbf{L}_{\sigma}^{\text {app }}\right]$ and $\left[\mathbf{L}_{\epsilon}^{\text {app }}\right]$. Using the same methodology as in Section 2.1.3 (that is, introducing a partitioning of $\Omega$ ), it can be shown that the following inequality holds for any given realization of the random media $[10]$ :

$$
\left[\widehat{\mathbf{L}}_{\sigma}^{\mathrm{app}}\right] \leq\left[\mathbf{L}_{\sigma}^{\mathrm{app}}\right] \leq\left[\mathbf{L}_{\epsilon}^{\mathrm{app}}\right] \leq\left[\widehat{\mathbf{L}}_{\epsilon}^{\mathrm{app}}\right]
$$

where $\left[\widehat{\mathbf{L}}_{\sigma}^{\text {app }}\right]$ and $\left[\widehat{\mathbf{L}}_{\epsilon}^{\text {app }}\right]$ are given by Eqs. (14-15). Again, taking a sufficiently large value of $n$ in Eq. (18) allows one to recover Reuss and Voigt-like bounds that are, unlike the usual Reuss and Voigt (deterministic) bounds, random (since mesoscopic domains are very likely to exhibit fluctuations in the volume fractions of the constituents, in addition to potential fluctuations 
of the elasticity tensors of the constitutive phases). Let $\left[\overline{\mathbf{L}^{-1}}\right]^{-1}$ and $[\overline{\mathbf{L}}]$ be these statistical Reuss and Voigt bounds.

The boundedness property (18) holds for apparent tensors defined with respect to very particular kinds of boundary conditions (namely, KUBC and SUBC) which may not be encountered in practical applications or may be tricky to reproduce experimentally (as pointed out in [8]). Thus, we now consider the more relevant case of mixed boundary conditions (MBC). The mechanical definition of the mixed apparent elasticity tensor in $\mathrm{MBC}$, denoted by $\left[\left[\mathbf{L}_{m}^{\mathrm{app}}\right]\right]$, then reads:

$$
<\sigma>=\left[\left[\mathbf{L}_{m}^{\mathrm{app}}\right]\right]:<\epsilon>
$$

Equivalently, one has:

$$
<\epsilon>=\left[\left[\mathbf{M}_{m}^{\mathrm{app}}\right]\right]:<\sigma>,
$$

where $\left[\left[\mathbf{M}_{m}^{\mathrm{app}}\right]\right]$ is the mixed apparent compliance tensor in MBC. Note that the above mechanical definitions do not coincide with the energetic ones, unless the retained boundary conditions satisfy the Hill condition (see [8] for a discussion). A particular family of MBC satisfying Eq. (6) is the one in which "uniform conditions are applied to any three of the six displacement or traction vector components" (this kind of boundary conditions may be referred to as orthogonal uniform mixed boundary conditions; see [8]), which includes the important practical case of uniaxial testing. In this context, one can further prove that [8]:

$$
\left[\mathbf{L}_{\sigma}^{\mathrm{app}}\right] \leq\left[\mathbf{L}_{m}^{\mathrm{app}}\right] \leq\left[\mathbf{L}_{\epsilon}^{\mathrm{app}}\right]
$$

so that:

$$
0<\left[\overline{\mathbf{L}^{-1}}\right]^{-1} \leq\left[\widehat{\mathbf{L}}_{\sigma}^{\mathrm{app}}\right] \leq\left[\mathbf{L}_{\sigma}^{\mathrm{app}}\right] \leq\left[\mathbf{L}_{m}^{\mathrm{app}}\right] \leq\left[\mathbf{L}_{\epsilon}^{\mathrm{app}}\right] \leq\left[\widehat{\mathbf{L}}_{\epsilon}^{\mathrm{app}}\right] \leq[\overline{\mathbf{L}}] .
$$

For any given realization of the random media, Eq. (22) provides an inequality between the corresponding realization of the apparent elasticity tensor of a domain smaller than the representative volume element, defined with respect to mixed boundary conditions (satisfying the Hill condition), and the associated realizations of apparent elasticity tensors derived, either by applying KUBC and SUBC (for $\left[\widehat{\mathbf{L}}_{\sigma}^{\mathrm{app}}\right],\left[\mathbf{L}_{\sigma}^{\mathrm{app}}\right],\left[\mathbf{L}_{\epsilon}^{\mathrm{app}}\right]$ and $\left[\widehat{\mathbf{L}}_{\epsilon}^{\mathrm{app}}\right]$ ) or without considering any boundary condition at all (for $\left[\overline{\mathbf{L}}^{-1}\right]^{-1}$ and $[\overline{\mathbf{L}}]$ ).

As will be discussed in Section 2.3, the boundedness constraint will be used for defining the support of the probability distribution and therefore, it should be pointed out that $\left[\widehat{\mathbf{L}}_{\sigma}^{\text {app }}\right]$ and $\left[\widehat{\mathbf{L}}_{\epsilon}^{\text {app }}\right]$ implicitly depend on the choice 
of the partitioning scheme, while $\left[\mathbf{L}_{\sigma}^{\text {app }}\right]$ and $\left[\mathbf{L}_{\epsilon}^{\text {app }}\right]$ do not (and are defined at the same scale as $\left.\left[\mathbf{L}_{m}^{\mathrm{app}}\right]\right)$.

It is worth pointing out that, in general, the apparent elasticity matrices in Eq. (22) are all random. For two-phase materials made up with ordered (in the positive-definite sense) phases (assume for instance that $\left[L^{(i)}\right]>\left[L^{(m)}\right]$, where $\left[L^{(m)}\right]$ and $\left[L^{(i)}\right]$ are the elasticity tensors of the matrix and inclusions, respectively), one can readily obtain lower and upper deterministic bounds setting:

$$
\begin{gathered}
{\left[L_{\ell}\right]=\left(\left(1-f_{\min }\right)\left[L^{(m)}\right]^{-1}+f_{\min }\left[L^{(i)}\right]^{-1}\right)^{-1},} \\
{\left[L_{u}\right]=\left(1-f_{\max }\right)\left[L^{(m)}\right]+f_{\max }\left[L^{(i)}\right],}
\end{gathered}
$$

in which $f_{\min } \in[0,1]$ and $f_{\max } \in[0,1]$ are the lower and upper bound of the compact support of the probability density function of the random volume fraction of phase $(i)$. In the most general case, scale-dependent deterministic bounds $\left[L_{\ell}\right]$ and $\left[L_{u}\right]$ have to be computed from a set of realizations of the statistical Reuss and Voigt bounds (or from a set of realizations of $\left[\mathbf{L}_{\sigma}^{\text {app }}\right]$ and $\left[\mathbf{L}_{\epsilon}^{\text {app }}\right]$, yielding slightly tighter bounds in view of Eq. (22)). Noticing that Loewner ordering is partial, it follows that supremum and infimum can not be defined on a set of realizations (unlike in the real, scalar-valued case) and a specific numerical strategy to circumvent this difficulty will be formulated in Section 4.3.1. Note finally that while such a choice clearly introduces some modeling bias, it is however consistent with the usual way to perform identification on uniform distribution with limited experimental data. Eq. (22) then reads:

$$
\left[L_{\ell}\right] \leq\left[\mathbf{L}_{m}^{\mathrm{app}}\right] \leq\left[L_{u}\right]
$$

almost surely (a.s.). Eq. (25) states that each realization of the random matrix $\left[\mathbf{L}_{m}^{\mathrm{app}}\right]$ is deterministically bounded from above and below. Such a boundedness constraint will be considered in the construction of the probabilistic model, as discussed in the next section.

\subsection{Overview of the construction}

With reference to the notations introduced in the previous section, let $\left[L_{\ell}\right] \in \mathbb{M}_{n}^{+}(\mathbb{R})$ and $\left[L_{u}\right] \in \mathbb{M}_{n}^{+}(\mathbb{R})$ be two given deterministic matrices. Let $[\mathbf{L}]$ be a $\mathbb{M}_{n}^{+}(\mathbb{R})$-valued random variable, defined on probability space $(\Theta, \mathcal{T}, \mathcal{P})$ and corresponding to a matrix representation of the apparent elasticity tensor $[[\mathbf{L}]]$. It is assumed that Eq. (25) holds in the strict sense. Let $[L] \mapsto p_{[\mathbf{L}]}([L])$ be the probability density function, from $\mathcal{S}=\{[L] \in$ 
$\left.\mathbb{M}_{n}^{+}(\mathbb{R}) \mid\left[L_{\ell}\right]<[L]<\left[L_{u}\right]\right\}$ into $\mathbb{R}^{+}$, defining the probability distribution $P_{[\mathbf{L}]}=p_{[\mathbf{L}]}([L]) d L$ of random matrix $[\mathbf{L}]$. The measure $d L$ on $\mathbb{M}_{n}^{S}(\mathbb{R})$ is defined as $d L=2^{n(n-1) / 4} \prod_{1 \leq i \leq j \leq n} d[L]_{i j}$, where $d[L]_{i j}$ is the Lebesgue measure on $\mathbb{R}$ (see [31]).

The measure of entropy, introduced for discrete probability distributions by Shannon [29], of the probability density function $p$ is defined as:

$$
\mathrm{S}(p)=-\int_{\mathbb{M}_{n}^{+}(\mathbb{R})} p([L]) \ln (p([L])) d L
$$

where $\ln$ is the Neperian logarithm. The Maximum Entropy principle, stated by Jaynes in the case of random vectors (see [13] [14]), consists in maximizing the measure of entropy $\mathrm{S}(p)$, under a set of constraints defining the available information. Such a principle basically allows one to construct a probabilistic model without introducing any bias in the estimation of the probability distribution. Thus, denoting by $\mathcal{C}_{a d}$ the set of all the probability density functions, with support $\mathcal{S}$, such that all the constraints defining the available information are fulfilled, the probability density function $p_{[\mathbf{L}]}$ constructed using the MaxEnt principle is such that:

$$
p_{[\mathbf{L}]}=\underset{p \in \mathcal{C}_{a d}}{\arg \max } \mathrm{S}(p) .
$$

Such a problem is classically solved by introducing a set of Lagrange multipliers and having recourse to the calculus of variations, applied to the resulting Lagragian. The set of constraints considered in this paper, as well as the resulting explicit form of the probability density function $p_{[\mathbf{L}]}$, are presented in the next section.

\subsection{Probabilistic models}

\subsubsection{Probabilistic model based on the generalized matrix variate Kummer-} Beta distribution

Let us first consider the following set of constraints:

$$
\begin{gathered}
\int_{\mathcal{S}} p_{[\mathbf{L}]}([L]) d L=1, \\
\mathrm{E}\{[\mathbf{L}]\}=[\underline{L}] \in \mathbb{M}_{n}^{+}(\mathbb{R}), \\
\mathrm{E}\left\{\ln \left(\operatorname{det}\left([\mathbf{L}]-\left[L_{\ell}\right]\right)\right)\right\}=\nu_{\ell}, \quad\left|\nu_{\ell}\right|<+\infty, \\
\mathrm{E}\left\{\ln \left(\operatorname{det}\left(\left[L_{u}\right]-[\mathbf{L}]\right)\right)\right\}=\nu_{u}, \quad\left|\nu_{u}\right|<+\infty,
\end{gathered}
$$


where $\mathrm{E}$ is the mathematical expectation. Eq. (28) is the usual normalization condition of the probability density function, while Eq. (29) means that the mean value of $[\mathbf{L}]$ is supposed to be known. Eqs. (30-31) are related to the boundedness constraint on [L] (see [31] for a detailed discussion). This set of constraints has already been considered in [2]. Let $\lambda_{0} \in \mathbb{R},\left[\Lambda_{[\mathbf{L}]}\right] \in \mathbb{M}_{n}^{+}(\mathbb{R}), \lambda_{\ell} \in \mathbb{R}$ and $\lambda_{u} \in \mathbb{R}$ be the real Lagrange multipliers corresponding to constraints (28), (29), (30) and (31), respectively. A straightforward derivation then yields:

$$
\begin{aligned}
p_{[\mathbf{L}]}([L])= & \mathbb{I}_{\mathcal{S}}([L]) c_{0} \operatorname{det}\left([L]-\left[L_{\ell}\right]\right)^{\lambda_{\ell}-1} \operatorname{det}\left(\left[L_{u}\right]-[L]\right)^{\lambda_{u}-1} \\
& \times \operatorname{etr}\left\{-\left[\Lambda_{[\mathbf{L}]}\right][L]\right\},
\end{aligned}
$$

in which $\operatorname{etr}\{[X]\})=\exp \{\operatorname{tr}([X])\}, c_{0}=\exp \left(-\lambda_{0}\right)$ is the normalization constant and $[X] \mapsto \mathbb{I}_{\mathcal{S}}([X])$ is the indicator function of $\mathcal{S}$. Assuming that the Lagrange multipliers $\lambda_{\ell}$ and $\lambda_{u}$ are both strictly positive, it is seen that Eq. (32) corresponds to the probability density function of a generalized matrix variate Kummer-Beta distribution [17], allowing a closed-form expression of $c_{0}$ to be derived.

When a sufficiently large number of realizations of $[\mathbf{L}]$ are available, the parameters $[\underline{L}], \nu_{\ell}$ and $\nu_{u}$ involved in Eqs. (29-31) can be estimated by the usual mathematical statistics [28]. In this context, an algorithmic scheme for computing the multipliers $\left[\Lambda_{[\mathbf{L}]}\right], \lambda_{\ell}$ and $\lambda_{u}$ is provided in [2].

\subsubsection{Alternative formulation}

By definition, the random matrix $[\mathbf{L}]$ is such that:

$$
\left[L_{\ell}\right]<[\mathbf{L}]<\left[L_{u}\right] \text { a.s. }
$$

Since $[\mathbf{L}]-\left[L_{\ell}\right] \in \mathbb{M}_{n}^{+}(\mathbb{R})$ a.s., the random matrix $\left([\mathbf{L}]-\left[L_{\ell}\right]\right)^{-1}$ exists a.s. and is such that $\left([\mathbf{L}]-\left[L_{\ell}\right]\right)^{-1} \in \mathbb{M}_{n}^{+}(\mathbb{R})$ a.s. Similarly, the deterministic matrix $\left(\left[L_{u}\right]-\left[L_{\ell}\right]\right)^{-1}$ is symmetric definite-positive. We then introduce the $\mathbb{M}_{n}^{+}(\mathbb{R})$-valued random variable $[\mathbf{N}]$, defined as:

$$
[\mathbf{N}]=\left([\mathbf{L}]-\left[L_{\ell}\right]\right)^{-1}-\left(\left[L_{u}\right]-\left[L_{\ell}\right]\right)^{-1} .
$$

It is readily seen that Eq. (33) is strictly equivalent to the positive-definiteness of $[\mathbf{N}]$, that is:

$$
0<[\mathbf{N}] \text { a.s. }
$$

Let $[N] \mapsto p_{[\mathbf{N}]}([N])$ be the probability density function of random matrix $[\mathbf{N}]$, defined on $\mathbb{M}_{n}^{+}(\mathbb{R})$. Let us now consider the following constraints:

$$
\int_{\mathbb{M}_{n}^{+}(\mathbb{R})} p_{[\mathbf{N}]}([N]) d N=1
$$




$$
\begin{gathered}
\mathrm{E}\{[\mathbf{N}]\}=\mathrm{E}\left\{\left([\mathbf{L}]-\left[L_{\ell}\right]\right)^{-1}\right\}-\left(\left[L_{u}\right]-\left[L_{\ell}\right]\right)^{-1}=[\underline{N}], \\
\mathrm{E}\{\ln (\operatorname{det}([\mathbf{N}]))\}=\nu, \quad|\nu|<+\infty .
\end{gathered}
$$

Consequently, $p_{[\mathbf{N}]}$ takes the form [31]:

$$
p_{[\mathbf{N}]}([N])=\mathbb{I}_{\mathbb{M}_{n}^{+}(\mathbb{R})}([N]) c_{0}^{*} \operatorname{det}([N])^{\lambda-1} \operatorname{etr}\left\{-\left[\Lambda_{[\mathbf{N}]}\right][N]\right\},
$$

in which $c_{0}^{*}$ is the normalization constant, $\lambda \in \mathbb{R}$ and $\left[\Lambda_{[\mathbf{N}]}\right] \in \mathbb{M}_{n}^{+}(\mathbb{R})$ are the Lagrange multipliers associated with Eqs. (38) and (37), respectively. When $\lambda$ is a positive integer, Eq. (39) is the probability density function of a Wishart distribution (see [1] [7]). The Lagrange multiplier $\left[\Lambda_{[\mathbf{N}]}\right]$ can be shown to be given by (see [31], Section 3.4.):

$$
\left[\Lambda_{[\mathbf{N}]}\right]=\frac{n-1+2 \lambda}{2}[\underline{N}]^{-1} .
$$

Let $\delta_{[\mathbf{N}]}$ be the parameter defined as $\delta_{[\mathbf{N}]}^{2}=\mathrm{E}\left\{\|[\mathbf{N}]-[\underline{N}]\|_{\mathrm{F}}^{2}\right\} /\|[\underline{N}]\|_{\mathrm{F}}^{2}$, where $\|\cdot\|_{\mathrm{F}}$ denotes the Frobenius (or Hilbert-Schmidt) norm, allowing the level of statistical fluctuations of random matrix $[\mathbf{N}]$ to be characterized. It can further be shown that $\delta_{[\mathbf{N}]}$ can be expressed as an function of $\lambda$ and $[\underline{N}]$ (see [31], Eq. (51)). From Eqs. (34) and (39), it can be deduced that the probability density function $[L] \mapsto p_{[\mathbf{L}]}([L])$ of random matrix $[\mathbf{L}]$ then reads:

$$
\begin{aligned}
p_{[\mathbf{L}]}([L])= & \mathbb{I}_{\mathbb{M}_{n}^{+}(\mathbb{R})}\left(\left([L]-\left[L_{\ell}\right]\right)^{-1}-\left(\left[L_{u}\right]-\left[L_{\ell}\right]\right)^{-1}\right) c_{0}^{* *} \\
& \times \operatorname{det}\left(\left([L]-\left[L_{\ell}\right]\right)^{-1}-\left(\left[L_{u}\right]-\left[L_{\ell}\right]\right)^{-1}\right)^{\lambda-1} \\
& \times \operatorname{det}\left([L]-\left[L_{\ell}\right]\right)^{-(n+1)} \operatorname{etr}\left\{-\left[\Lambda_{[\mathbf{N}]}\right]\left([L]-\left[L_{\ell}\right]\right)^{-1}\right\},
\end{aligned}
$$

in which $c_{0}^{* *}$ is a new normalization constant. In terms of random generation, once a sample of $[\mathbf{N}]$ has been drawed w.r.t. Eq. (39), the corresponding realization of $[\mathbf{L}]$ is readily computed from Eq. (34).

The above probabilistic models are now briefly to be discussed in the next section.

\subsubsection{Discussion}

Clearly, the two probabilistic models mainly differ in the way the boundedness constraint is introduced. In Section 2.3.1, the constraint is formalized in its natural form, that is, w.r.t random matrix $[\mathbf{L}]$, yielding the final form (32) of the probability density function $[L] \mapsto p_{[\mathbf{L}]}([L])$. Such a strategy allows the mean value of $[\mathbf{L}]$ to be explicitly constrained and offers more flexibility in prescribing the behavior of the distribution around the bounds (through the parameters $\lambda_{\ell}$ and $\lambda_{u}$ ). Conceptually, the methodology followed in Section 2.3.2 consists in constructing a probabilistic model for the 
"shifted inverse" random matrix $[\mathbf{N}]$, while using information available for $[\mathbf{L}]$. Consequently, such a strategy does not allow constraining the mean value of $[\mathbf{L}]$, at least in a direct way. Note however that in this case, the same amount of information is taken into account through the use of realizations of random matrix $[\mathbf{L}]$.

From a numerical standpoint, the generation of random matrix $[\mathbf{L}]$ w.r.t. Eq. (32) requires the use of either Markov Chain Monte Carlo methods (such as Gibbs sampling or the Metropolis algorithm) or the slice sampling technique [18] (as discussed in [2]). On the contrary, the generation of realizations of random matrix [N] (w.r.t. Eq. (39)) relies on Monte-Carlo simulations of Gaussian and gamma univariate random variables (see the numerical strategy proposed in [31]). Consequently, the second approach relies on a more robust generator and outperforms the first one in terms of computational cost. More importantly, the alternative formulation in Section 2.3.2 further allows the probabilistic model to be readily extended to the case of $\mathbb{M}_{n}^{+}(\mathbb{R})$-valued random fields. Such an extension is addressed below.

\section{Probabilistic model for matrix-valued random field}

\subsection{Model derivation}

Let $\mathbf{x} \mapsto[\mathbf{L}(\mathbf{x})]$ be the $\mathbb{M}_{n}^{+}(\mathbb{R})$-valued random field defined on probability space $(\Theta, \mathcal{T}, \mathcal{P})$, indexed by a bounded open domain $\mathcal{D}$ in $\mathbb{R}^{d}$ ( $d$ being a positive integer, $1 \leq d \leq 3$ ) and corresponding to a suitable random matrix representation of the tensor-valued random field $\mathbf{x} \mapsto[[\mathbf{L}(\mathbf{x})]]$. The objective of this section is the construction of a prior stochastic model of matrixvalued random field $[\mathbf{L}]$ using only the available information and Information Theory. Therefore, the model thus obtained is related to this framework.

Following Section 2, we assume that there exists two given deterministic fields $\mathbf{x} \mapsto\left[L_{\ell}(\mathbf{x})\right]$ and $\mathbf{x} \mapsto\left[L_{u}(\mathbf{x})\right]$ (which are intrinsically scale-dependent; see the discussion in Section 2.1$)$, with values in $\mathbb{M}_{n}^{+}(\mathbb{R})$, such that:

$$
0<\left[L_{\ell}(\mathbf{x})\right] \leq[\mathbf{L}(\mathbf{x})] \leq\left[L_{u}(\mathbf{x})\right]
$$

for $\mathbf{x}$ fixed in $\mathcal{D}$. Let $\mathbf{x} \mapsto[\mathbf{N}(\mathbf{x})]$ be the $\mathbb{M}_{n}^{+}(\mathbb{R})$-valued random field defined on probability space $(\Theta, \mathcal{T}, \mathcal{P})$, indexed by $\mathcal{D}$, such that for all $\mathbf{x}$ in $\mathcal{D}$ :

$$
[\mathbf{N}(\mathbf{x})]=\left([\mathbf{L}(\mathbf{x})]-\left[L_{\ell}(\mathbf{x})\right]\right)^{-1}-\left(\left[L_{u}(\mathbf{x})\right]-\left[L_{\ell}(\mathbf{x})\right]\right)^{-1} .
$$

It is assumed that the mean function $\mathbf{x} \mapsto \mathrm{E}\{[\mathbf{N}(\mathbf{x})]\}=[\underline{N}(\mathbf{x})]$, defined from $\mathcal{D}$ into $\mathbb{M}_{n}^{+}(\mathbb{R})$, is given. This mean function can be either directly 
estimated using Eq. (43) when realizations of $[\mathbf{L}(\mathbf{x})]$ are available or in the most general case, computed solving an inverse (boundary value) problem. It can then be deduced that there exists a deterministic field $\mathbf{x} \mapsto[\underline{M}(\mathbf{x})]$, where $[\underline{M}(\mathbf{x})]$ is an invertible upper triangular matrix, such that:

$$
[\underline{N}(\mathbf{x})]=[\underline{M}(\mathbf{x})]^{\mathrm{T}}[\underline{M}(\mathbf{x})],
$$

for $\mathbf{x} \in \mathcal{D}$. We assume that the available information is the one considered in [33] [34] and then, the prior stochastic model of random field $\mathbf{x} \mapsto[\mathbf{N}(\mathbf{x})]$ belongs to the class defined in these references. For $\mathbf{x}$ fixed in $\mathcal{D}$, the random matrix $[\mathbf{N}(\mathbf{x})]$ is thus written as:

$$
[\mathbf{N}(\mathbf{x})]=[\underline{M}(\mathbf{x})]^{\mathrm{T}}[\mathbf{K}(\mathbf{x})][\underline{M}(\mathbf{x})],
$$

in which $\mathbf{x} \mapsto[\mathbf{K}(\mathbf{x})]$ is a homogeneous non-Gaussian second-order $\mathbb{M}_{n}^{+}(\mathbb{R})$ valued random field, indexed by $\mathbb{R}^{d}$, defined on probability space $(\Theta, \mathcal{T}, \mathcal{P})$, such that $\mathrm{E}\{[\mathbf{K}(\mathbf{x})]\}=\left[I_{n}\right]\left(\left[I_{n}\right]\right.$ being the $(n \times n)$ identity matrix $)$. Let $\delta_{[\mathbf{K}}$ be defined as:

$$
\delta_{[\mathbf{K}]}^{2}=\frac{1}{n^{2}} \mathrm{E}\left\{\left\|[\mathbf{K}(\mathbf{x})]-\left[I_{n}\right]\right\|_{\mathrm{F}}^{2}\right\}
$$

with $0<\delta_{[\mathbf{K}]}<\sqrt{(n+1) /(n+5)}$. The random field $\mathbf{x} \mapsto[\mathbf{K}(\mathbf{x})]$ is defined by a nonlinear mapping of $n(n+1) / 2$ independent second-order centered homogeneous $\mathbb{R}$-valued Gaussian random fields, denoted by $\mathbf{x} \mapsto \zeta_{i j}(\mathbf{x})$ for $1 \leq i \leq j \leq n$, defined on $(\Theta, \mathcal{T}, P)$ and indexed by $\mathbb{R}^{d}$. Specifically, for $\mathbf{x}$ fixed in $\mathcal{D}$, the random matrix $[\mathbf{K}(\mathbf{x})]$ is written as:

$$
[\mathbf{K}(\mathbf{x})]=[\mathbf{P}(\mathbf{x})]^{\mathrm{T}}[\mathbf{P}(\mathbf{x})],
$$

in which $[\mathbf{P}(\mathbf{x})]$ is a upper triangular matrix defined by:

$$
[\mathbf{P}(\mathbf{x})]_{i j}=\frac{\delta_{[\mathbf{K}]}}{\sqrt{n+1}} \zeta_{i j}(\mathbf{x})
$$

for $i<j$ and:

$$
[\mathbf{P}(\mathbf{x})]_{i i}=\frac{\delta_{[\mathbf{K}]}}{\sqrt{n+1}} \sqrt{2 h_{a_{i}}\left(\zeta_{i i}(\mathbf{x})\right)},
$$

in which $a_{i}=(n+1) /\left(2 \delta_{[\mathbf{K}]}^{2}\right)+(1-i) / 2$ and $u \mapsto h_{a_{i}}(u)$ is the function parameterized by $a_{i}>0$ and defined from $\mathbb{R}$ into $\mathbb{R}^{+}$by:

$$
h_{a_{i}}(u)=F_{\Gamma_{a_{i}}}^{-1}\left(F_{U}(u)\right),
$$

where $u \mapsto F_{U}(u)$ is the cumulative distribution function of the normalized Gaussian random variable $U$ and $p \mapsto F_{\Gamma_{a_{i}}}^{-1}(p)$ is the reciprocal function of 
the cumulative distribution function $\gamma \mapsto F_{\Gamma_{a_{i}}}(\gamma)$ of the Gamma random variable $\Gamma_{a_{i}}$ with parameter $a_{i}$. A set of further fundamental properties of random fields $\mathbf{x} \mapsto[\mathbf{K}(\mathbf{x})]$ and $\mathbf{x} \mapsto[\mathbf{N}(\mathbf{x})]$ can be found in [33] [34] (note that the mapping will be described, from an algorithmic standpoint, in Section 3.2). It can finally be shown that:

$$
\begin{aligned}
\delta_{[\mathbf{N}]}(\mathbf{x}) & =\left\{\mathrm{E}\left\{\|[\mathbf{N}(\mathbf{x})]-[\underline{N}(\mathbf{x})]\|_{\mathrm{F}}^{2}\right\} /\|[\underline{N}(\mathbf{x})]\|_{\mathrm{F}}^{2}\right\}^{1 / 2} \\
& =\frac{\delta_{[\mathbf{K}]}}{\sqrt{n+1}}\left\{1+\frac{(\operatorname{tr}[\underline{N}(\mathbf{x})])^{2}}{\operatorname{tr}\left([\underline{N}(\mathbf{x})]^{2}\right)}\right\}^{1 / 2} .
\end{aligned}
$$

Each random field of the family of stochastic germs $\left\{\mathbf{x} \mapsto \zeta_{i j}(\mathbf{x})\right\}_{i, j}$ is completely defined by its correlation function $\mathbf{y} \in \mathbb{R}^{d} \mapsto R_{\zeta_{i j}}(\mathbf{y})=\mathrm{E}\left\{\zeta_{i j}(\mathbf{x}+\right.$ $\left.\mathbf{y}) \zeta_{i j}(\mathbf{x})\right\}$, such that:

$$
R_{\zeta_{i j}}(\mathbf{y})=\prod_{k=1}^{d} r_{\zeta_{i j}}^{k}\left(y_{k}\right),
$$

in which $r_{\zeta_{i j}}^{k}\left(y_{k}\right)=\left(2 l_{i j}^{k} /\left(\pi y_{k}\right)\right)^{2} \sin ^{2}\left(\pi y_{k} /\left(2 l_{i j}^{k}\right)\right)$ for $k=1, \ldots, d$. The parameter $l_{i j}^{k}$ corresponds to the spatial correlation length of random field $\mathbf{x} \mapsto \zeta_{i j}(\mathbf{x})$ in the direction identified by index $k$. The correlation structure is thus entirely defined by the $d n(n+1) / 2$ correlation lengths of the Gaussian germs, ensuring a minimal parametrization of the probabilistic model.

Following [33], $\mathbf{x} \mapsto[\mathbf{N}(\mathbf{x})]$ is a second-order random field, that is to say,

$$
\mathrm{E}\left\{\|[\mathbf{N}(\mathbf{x})]\|_{\mathrm{F}}^{2}\right\}<+\infty,
$$

for all $\mathbf{x}$ in $\mathcal{D}$, with continuous realizations on the closure $\overline{\mathcal{D}}$ of $\mathcal{D}$ a.s. (provided that $\mathbf{x} \mapsto[\underline{N}(\mathbf{x})]$ is continuous on $\overline{\mathcal{D}})$. Consequently, it can be deduced, taking into account the results provided in [33], that $\mathbf{x} \mapsto[\mathbf{L}(\mathbf{x})]$ is a second-order random field on $\mathcal{D}$. Having recourse to such a construction, the probabilistic model for $\mathbf{x} \mapsto[\mathbf{L}(\mathbf{x})]$ is then completely defined by:

(i) The mean function $\mathbf{x} \mapsto[\underline{N}(\mathbf{x})]$.

(ii) The field $\mathbf{x} \mapsto \delta_{[\mathbf{N}]}(\mathbf{x})$ (or equivalently, $\delta_{[\mathbf{K}]}$; see Eq. (51)).

(iii) The set of spatial correlation lengths $\left\{l_{i j}^{k}\right\}, 1 \leq i \leq j \leq n, k=1, \ldots, d$.

(iv) The deterministic fields $\mathbf{x} \mapsto\left[L_{\ell}(\mathbf{x})\right]$ and $\mathbf{x} \mapsto\left[L_{u}(\mathbf{x})\right]$.

When the mean function does not depend on $\mathbf{x}$, it can be deduced that $\delta_{[\mathbf{N}]}(\mathbf{x})=\delta_{[\mathbf{N}]}$ (see Eq. (51)) and $\mathbf{x} \mapsto[\mathbf{N}(\mathbf{x})]$ can then be seen as the 
restriction to domain $\mathcal{D}$ of a homogeneous random field indexed by $\mathbb{R}^{d}$. Furthermore, if the bounding fields $\mathbf{x} \mapsto\left[L_{\ell}(\mathbf{x})\right]$ and $\mathbf{x} \mapsto\left[L_{u}(\mathbf{x})\right]$ are constant (i.e. $\forall \mathbf{x} \in \mathcal{D},\left[L_{\ell}(\mathbf{x})\right]=\left[L_{\ell}\right]$ and $\left.\left[L_{u}(\mathbf{x})\right]=\left[L_{u}\right]\right)$, random field $\mathbf{x} \mapsto[\mathbf{L}(\mathbf{x})]$ can also be viewed as the restriction to domain $\mathcal{D}$ of a homogeneous random field indexed by $\mathbb{R}^{d}$.

\subsection{Implementation}

The main algorithm allowing realizations of random field $\mathbf{x} \mapsto[\mathbf{L}(\mathbf{x})]$ to be simulated is presented below. The numerical simulation of the Gaussian germs can be performed using the classical method introduced in [30] and is not detailed for the sake of brevity (note that the spatial correlation lengths are then implicit inputs of the algorithm).

input : $n, \delta_{[\mathbf{K}]}, N_{s i m},\left[L_{\ell}(\mathbf{x})\right],\left[L_{u}(\mathbf{x})\right],[\underline{M}(\mathbf{x})]$

output: $N_{\text {sim }}$ realizations of random field $\mathbf{x} \mapsto[\mathbf{L}(\mathbf{x})]$

$1 b \leftarrow \delta_{[\mathbf{K}]}(n+1)^{-1 / 2}$;

2 for $i \leftarrow 1, n$ do

$\mathbf{3} \mid a_{i} \leftarrow(n+1) /\left(2 \delta_{[\mathbf{K}]}^{2}\right)+(1-i) / 2$

4 end

$\mathbf{5}$ for $p \leftarrow 1, N_{\text {sim }}$ do

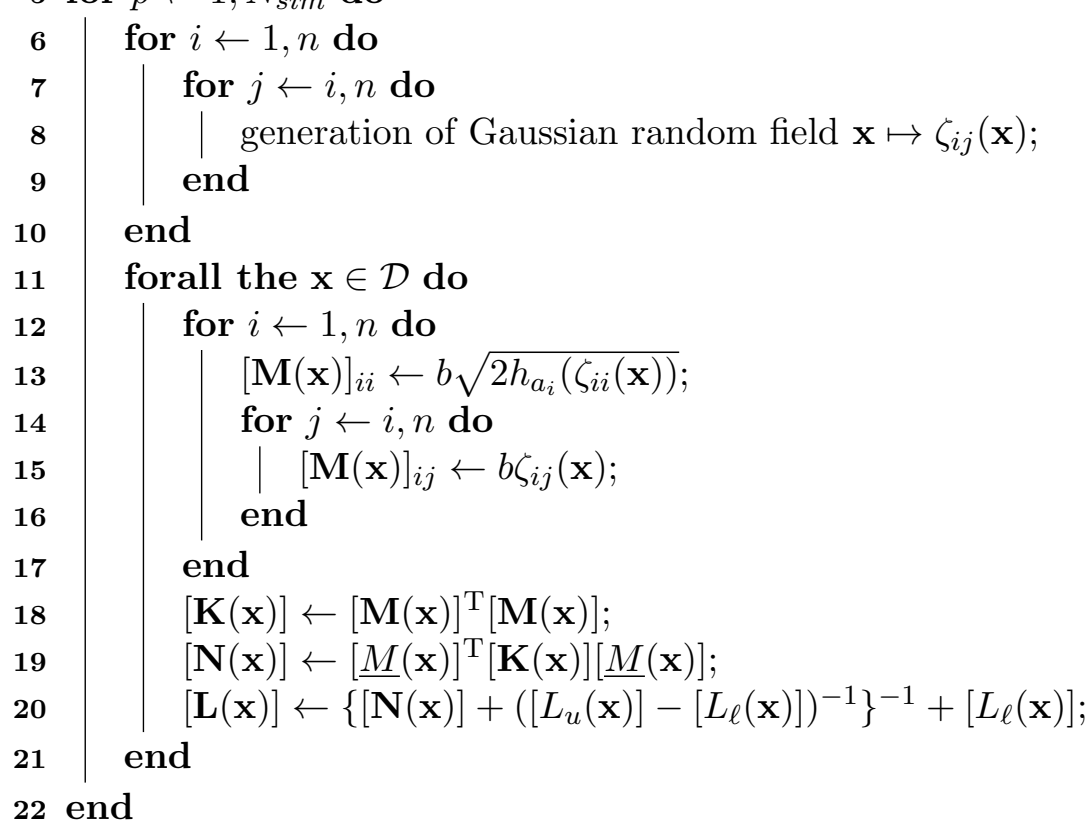

Algorithm 1: Numerical Monte Carlo simulations of random field. 


\section{Application}

\subsection{Experimental database}

As an application, we consider the case of an Al alloy (with commercial reference $\mathrm{Al}$ 2024-T351). Details about the experimental analysis can be found in [22]. The microstructural-crystallographic characterization is obtained using the EBSD technique (see [27] for instance). Specifically, measurements were obtained from a $6.35[\mathrm{~mm}]$ thick rolled plate, the faces of which were polished on the rolling plane as well as on the plan perpendicular to the rolling direction. Nine slightly overlapping $2 \times 1[\mathrm{~mm}]$ maps of the microstructure were obtained on each plane and only the data relative to the rolling plane are considered in this research. An experimental realization of the polycrystalline microstructure is shown on Fig. 1, where colors refer to crystallographic orientations.

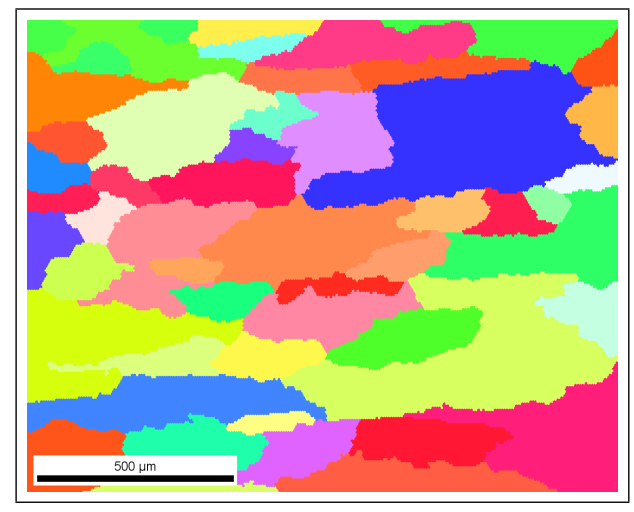

Figure 1: Experimental realization of the polycrystalline microstructure.

For characterization purposes, each grain $\mathcal{G}_{i}$ is fitted to an ellipse $\mathcal{E}_{i}$ (using a conic representation and a least-square estimation), so that the microstructure is classically described by the equivalent aspect ratios $\omega$ and Euler angles of all the grains. Let $\Phi^{i}=\left[\phi_{1}^{i}, \phi^{i}, \phi_{3}^{i}\right]$ be the random vector of Euler angles corresponding to grain $\mathcal{G}_{i}$. For a given realization of the microstructure containing, say, $N_{\mathcal{G}}$ grains, the $N_{\mathcal{G}}$ realizations of $\omega$ and $\Phi$ are considered as independent realizations and thus, the experimental database is made up of 394 independent realizations of $\omega$ and $\Phi$. The kernel estimate of the probability density function of $\omega$ is plotted in Fig. 2. It is seen that most of the realizations of the aspect ratio are significantly different from 
1, so that most of the grains appear elongated. Furthermore, the angular definition of the axis characterizing the fitting ellipses shows that the grains are all elongated in the same direction defined by, say, vector $\mathbf{e}_{1}$ (see also Fig. 1). Note that this feature is typically encountered for the kind of alloy considered in this research and does not result from the rolling process. The plot of the probability density functions of the three Euler angles is shown in Fig. 3. It is seen that angles $\phi_{1}$ and $\phi_{3}$ are, roughly speaking, uniformly distributed over $[0,2 \pi]$, while $\phi$ has a slightly bimodal distribution supported over $[0, \pi / 2]$.

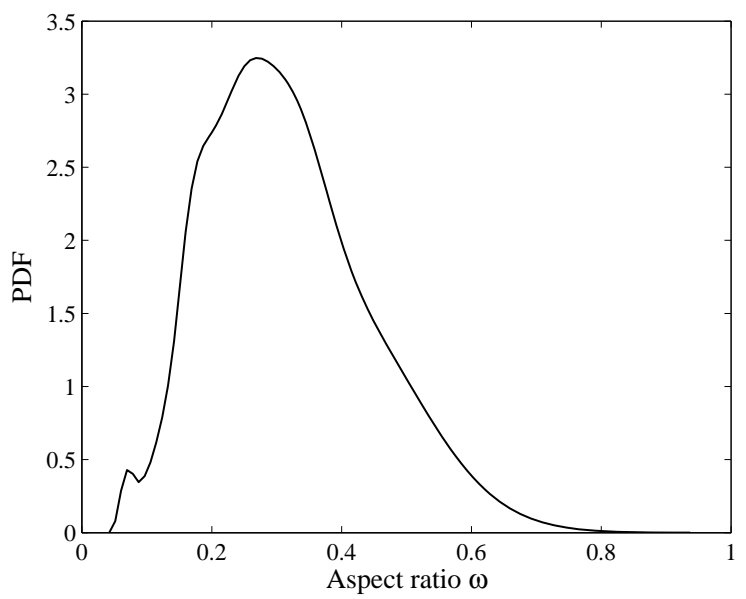

Figure 2: Plot of the probability density function of $\omega$.

\subsection{Simulation of a database}

This section aims at defining a general framework for digitally simulating 2D microstructures in accordance with the experimental data previously defined. Such simulations will be latter used to ensure a reasonable level of convergence in the identification of the probabilistic model. From Section 4.1 , it turns out that the numerical simulation of the polycrystalline microstructure relies on the simulation of both the grain geometry and the crystallographic orientation parameterized by the Euler angles.

The simulation of the crystallographic orientation involves sampling from the joint distribution of $\phi_{1}, \phi$ and $\phi_{3}$ and is performed in practice prescribing the marginal cumulative distribution functions (estimated from the experimental data) and the spearman's rank correlation matrix (see [11] for 


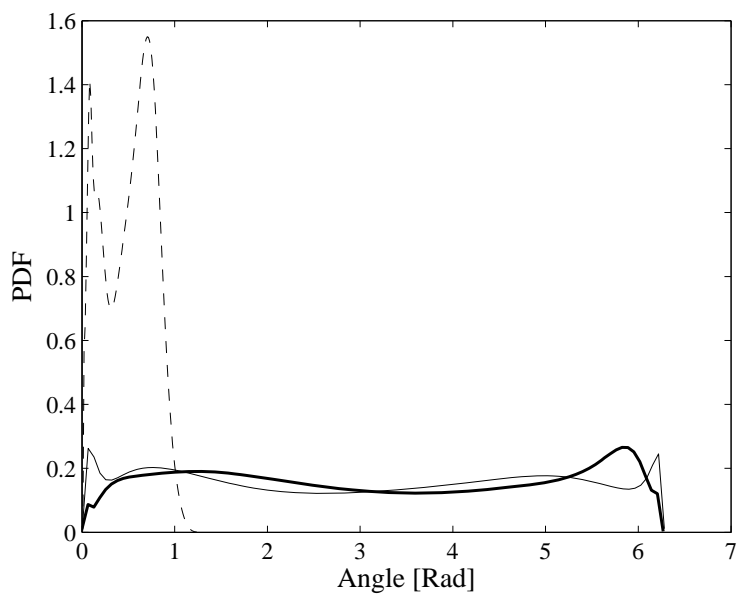

Figure 3: Plot the probability density functions of $\phi_{1}$ (thick solid line), $\phi$ (dashed line) and $\phi_{3}$ (thin solid line).

instance).

Voronoi tessellation [20] being a very common way to model the polycrystalline microstructure in metallic alloys, a first natural approach to simulate the grain geometry would then consist in using a two-dimensional Voronoi tessellation, typically generated from a homogeneous Poisson point process and as such, usually referred to as a Poisson-Voronoi tessellation. At this stage, it should however be pointed out that such a partitioning technique is not able to generate cells that are all elongated in a given direction and thus, it may introduce a modeling bias in the identification process. This difficulty can be circumvented having recourse to the Voronoi-G tessellation, introduced in [26] and briefly reviewed hereafter. Let $\Omega \subset \mathbb{R}^{d}$ be a bounded open set. Let $\left\{\mathbf{x}_{\text {tes }}^{(i)}\right\}_{i=1}^{N_{\text {tes }}}$ be the set of generating points belonging to the closure $\bar{\Omega}$ of $\Omega$. The cell $\mathcal{V}\left(\mathbf{x}_{\text {tes }}^{(i)}\right)$ generated by $\mathbf{x}_{\text {tes }}^{(i)}$ is the set of all the points of $\Omega$ that are closer to $\mathbf{x}_{\text {tes }}^{(i)}$ than any other generating point, the closeness being defined with respect to the Euclidean distance in $\mathbb{R}^{d}$. The set $\left\{\mathcal{V}\left(\mathbf{x}_{\text {tes }}^{(i)}\right)\right\}_{i=1}^{N_{\text {tes }}}$ is then referred to as a Voronoi tessellation (or Voronoi diagram) of $\Omega$. For $(\mathbf{x}, \mathbf{y}) \in \mathbb{R}^{d} \times \mathbb{R}^{d}$, let $(\mathbf{x}, \mathbf{y}) \mapsto d_{G}(\mathbf{x}, \mathbf{y})$ be the distance defined by:

$$
d_{G}(\mathbf{x}, \mathbf{y})=\sqrt{(\mathbf{x}-\mathbf{y})^{\mathrm{T}}[G](\mathbf{x}-\mathbf{y})}
$$

in which $[G]$ is a positive definite symmetric matrix. The cell $\mathcal{V}_{G}\left(\mathbf{x}_{\text {tes }}^{(i)}\right)$ of 
the Voronoi-G tessellation, generated by point $\mathbf{x}_{\text {tes }}^{(i)}$, is defined as:

$$
\mathcal{V}_{G}\left(\mathbf{x}_{\text {tes }}^{(i)}\right)=\left\{\mathbf{x} \in \Omega \mid d_{G}\left(\mathbf{x}_{\text {tes }}^{(i)}, \mathbf{x}\right) \leq d_{G}\left(\mathbf{x}_{\text {tes }}^{(j)}, \mathbf{x}\right)\right\},
$$

in which $j=1, \ldots, N_{\text {tes }}, j \neq i$. From Eq. (54), it is readily seen that setting $[G]=\left[I_{d}\right]$ allows one to recover the classical Voronoi tessellation. Let $[G]$ be defined as:

$$
[G]=\left[\begin{array}{cc}
1 / s^{2} & 0 \\
0 & 1
\end{array}\right]
$$

in which $s \in \mathbb{R}_{*}^{+}$. It can then be shown that the form (56) allows one to generate elongated cells in the $\mathbf{e}_{1}$-direction, parameter $s$ being interpreted as the rate of growth of the cell in this given direction. Thus, $1 / s$ may be seen as an overall aspect ratio and may be identified from the experimental distribution (see Fig. 2), enforcing an estimate $\widehat{s}$ of $s$ to be equal to the inverse of the aspect ratio empirical mean value for instance. However, it should be kept in mind that the scaling is applied to all the cells of a given realization of the microstructure, so that such an approach may, in some sense, over-constrain the numerical simulation. Subsequently, an estimate $\widehat{s}$ of parameter $s$ is here obtained from the experimental data using the Maximum Likelihood Approach and is then defined as:

$$
\widehat{s}=\underset{b \in \mathbb{R}_{*}^{+}}{\arg \max } \mathcal{L}\left(\omega_{\text {exp }}^{(1)}, \ldots, \omega_{\text {exp }}^{(394)}, b\right),
$$

in which the Log-likelihood function is defined as:

$$
\mathcal{L}\left(\omega_{\text {exp }}^{(1)}, \ldots, \omega_{\text {exp }}^{(394)}, b\right)=\sum_{i=1}^{394} \log \left(p_{\omega}\left(\omega_{\text {exp }}^{(i)}, b\right)\right),
$$

where $\log$ is the Neperian logarithm, $x \mapsto p_{\omega}(x, b)$ is the probability density function of the aspect ratio, estimated from numerical Monte Carlo simulations of the microstructure using Voronoi-G tessellation (setting $s=b$ ) and $\left\{\omega_{\text {exp }}^{(i)}\right\}_{i=1}^{394}$ are the experimental realizations of the aspect ratio. The plot of the Log-likelihood function, reported in Fig. 4, shows that the maximum is reached for $\widehat{s}=2.5$.

An example of microstructure, obtained before and after stretching, is shown on Fig. 5.

The algorithm used for generating the Voronoi-G tesselation is presented below (see [26]). 


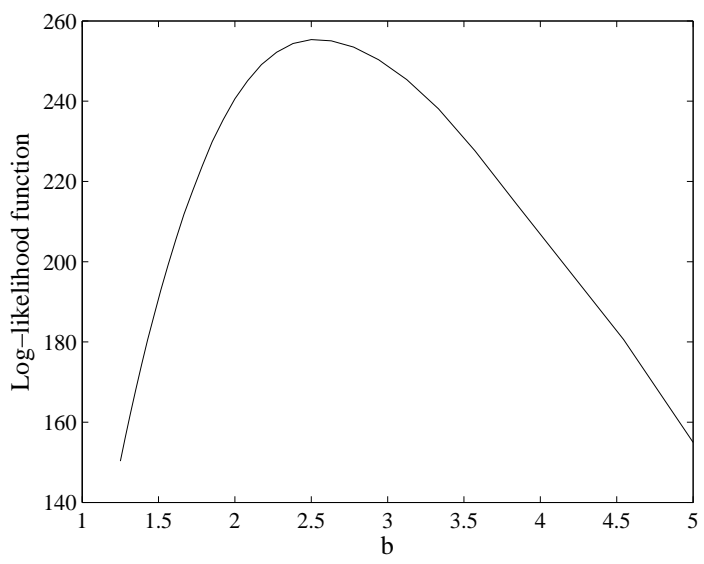

Figure 4: Plot of the likelihood function $b \mapsto \mathcal{L}\left(\omega_{\text {exp }}^{(1)}, \ldots, \omega_{\text {exp }}^{(394)}, b\right)$.
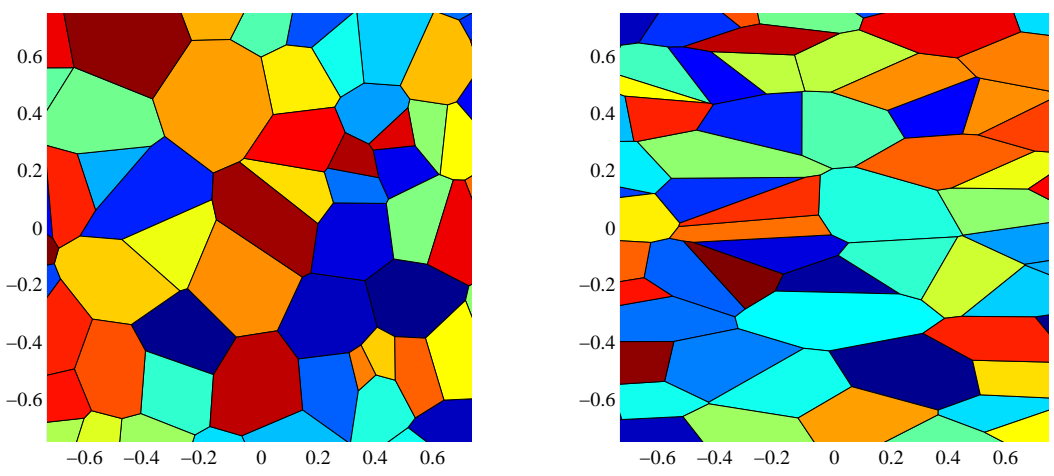

Figure 5: Example of digitally simulated microstructure, without (left) and with (right) elongation.

\subsection{Identification of the probabilistic model}

This section is devoted to the identification of the probabilistic model for random matrix $[\mathbf{N}]$. In particular, we do not address the identification of the parameters for random field $\mathbf{x} \mapsto[\mathbf{N}(\mathbf{x})]$, taking into account the limited amount of experimental data. Subsequently, we identify parameters $[\underline{N}]$ and $\delta_{[\mathbf{N}]}$ for a given mesoscopic domain. The correlation structure (that are, the set of spatial correlation lengths) will be assumed for illustration purposes in Section 4.4. 


$$
\begin{aligned}
& \text { input : }[G], \Omega \\
& \text { output: Voronoi-G tessellation of } \Omega
\end{aligned}
$$

$1[Q] \leftarrow[G]=[Q]^{\mathrm{T}}[Q]$

2 generate the generating points $\mathbf{x}_{\text {tes }}^{(i)} \in \Omega, i=1, \ldots, N_{\text {tes }}$;

3 for $i \leftarrow 1, N_{\text {tes }}$ do

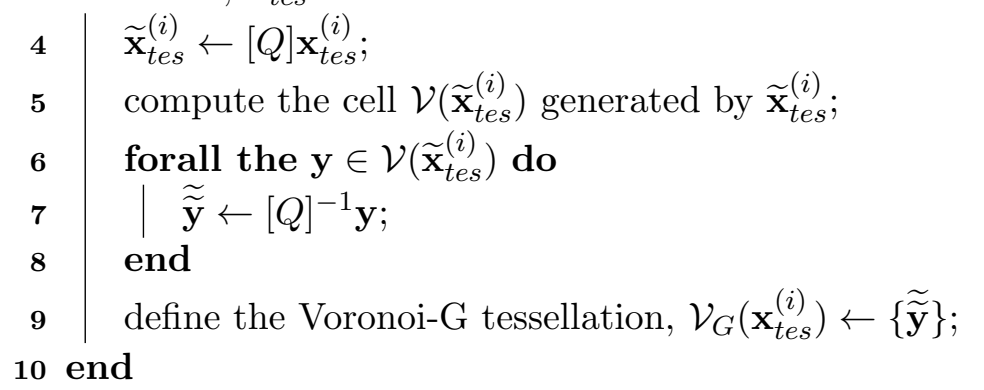

Algorithm 2: Construction of the Voronoi-G tessellation.

\subsubsection{Numerical strategy for computing the realizations of the apparent ten- sors}

The first step consists in computing a set of realizations of the bounds $\left[\widehat{\mathbf{L}}_{\sigma}^{\mathrm{app}}\right]$ and $\left[\widehat{\mathbf{L}}_{\epsilon}^{\mathrm{app}}\right]$, according to Sections 2.1.3 and 2.1.4. Let $N_{\text {sim }}$ be the number of simulated realizations of the microstructure. For this purpose, following Huet's Partition Theorem, domain $\Omega$ is divided into 9 subdomains $V_{i}$, for which the apparent tensors $\left[\mathbf{M}_{\sigma}^{\text {app }}\left(V_{i}\right)\right]$ and $\left[\mathbf{L}_{\epsilon}^{\text {app }}\left(V_{i}\right)\right]$ have to be computed considering both KUBC and SUBC. For a simulated realization of the polycrystalline microstructure and for a given kind of boundary condition $B C$, the mean stress $\langle\sigma\rangle_{B C}$ and mean strain $\langle\epsilon\rangle_{B C}$ vectors are computed averaging over $V_{i}$, the local stress and strain fields being computed using a Finite Element analysis. From a numerical standpoint, it can be verified that $\left\langle\epsilon>_{K U B C} \approx \epsilon_{0}\right.$ and that $\left\langle\sigma>_{S U B C} \approx \sigma_{0}\right.$. The corresponding realizations of $\left[\mathbf{L}_{\epsilon}^{\mathrm{app}}\left(V_{i}\right)\right]$ and $\left[\mathbf{M}_{\sigma}^{\mathrm{app}}\left(V_{i}\right)\right]$ are then defined as:

$$
\begin{gathered}
{\left[L_{\epsilon}^{\mathrm{app}}\left(V_{i}\right)\right]=\underset{\left[\overline{L^{-1}}\right]^{-1}<[L]<[\bar{L}]}{\arg \min }\left\|<\sigma>_{K U B C}-[L]<\epsilon>_{K U B C}\right\|,} \\
{\left[M_{\sigma}^{\mathrm{app}}\left(V_{i}\right)\right]=\underset{[\bar{L}]^{-1}<[M]<\left[\overline{L^{-1}}\right]}{\arg \min }\left\|<\sigma>_{S U B C}-[M]^{-1}<\epsilon>_{S U B C}\right\|,}
\end{gathered}
$$

in which $\|\cdot\|$ is any suitable norm, $\left[\overline{L^{-1}}\right]$ and $[\bar{L}]$ are respectively defined as:

$$
\left[\overline{L^{-1}}\right]=\sum_{k=1}^{N_{\mathcal{G}}} \frac{\left|\mathcal{G}_{k}\right|}{\left|V_{i}\right|}\left[L_{k}\right]^{-1}
$$




$$
[\bar{L}]=\sum_{k=1}^{N_{\mathcal{G}}} \frac{\left|\mathcal{G}_{k}\right|}{\left|V_{i}\right|}\left[L_{k}\right],
$$

where $N_{\mathcal{G}}$ is the realization of the random variable corresponding to the number of grains, $\left|\mathcal{G}_{k}\right|$ and $\left[L_{k}\right]$ are the area and the realization of the elasticity tensor of grain $\mathcal{G}_{k}$. The general form of the cost function in Eqs. (59-60) has been proposed in [2]. These equations are solved having recourse to semidefinite programming [39] (see also [3] Chapter 4) and more precisely, using the MATLAB toolbox YALMIP [15]. Once the realizations of $\left[\mathbf{L}_{\epsilon}^{\text {app }}\left(V_{i}\right)\right]$ and $\left[\mathbf{M}_{\sigma}^{\text {app }}\left(V_{i}\right)\right], i=1, \ldots, 9$, have been computed, the associated realizations $\left[\widehat{L}_{\sigma}^{\mathrm{app}}\right]$ and $\left[\widehat{L}_{\epsilon}^{\mathrm{app}}\right]$ of $\left[\widehat{\mathbf{L}}_{\sigma}^{\mathrm{app}}\right]$ and $\left[\widehat{\mathbf{L}}_{\epsilon}^{\mathrm{app}}\right]$ are estimated using Eqs. (14) and (15) respectively.

The second step of the methodology deals with the computation of the realization of the apparent elasticity matrices $\left[\mathbf{L}_{\epsilon}^{\mathrm{app}}\right]$ and $\left[\mathbf{L}_{\sigma}^{\mathrm{app}}\right]$. These realizations can be similarly computed applying KUBC and SUBC to the whole domain $\Omega$ and are defined as follows:

$$
\begin{aligned}
{\left[L_{\epsilon}^{\mathrm{app}}\right] } & =\underset{\left[\widehat{L}_{\sigma}^{\mathrm{app}}\right]<[L]<\left[\widehat{L}_{\epsilon}^{\mathrm{app}}\right]}{\arg \min }\left\|<\sigma>_{K U B C}-[L]<\epsilon>_{K U B C}\right\|, \\
{\left[L_{\sigma}^{\mathrm{app}}\right] } & =\underset{\left[\widehat{L}_{\sigma}^{\mathrm{app}}\right]<[L]<\left[\widehat{L}_{\epsilon}^{\mathrm{app}}\right]}{\arg \min }\left\|<\sigma>_{S U B C}-[L]<\epsilon>_{S U B C}\right\| .
\end{aligned}
$$

The final step involves the computation of the realizations of the apparent elasticity matrix $\left[\mathbf{L}_{m}^{\text {app }}\right]$ for mixed boundary conditions (a tensile test here). In order to be consistent with Eq. (25), such calculations first require the estimates of the deterministic bounds $\left[L_{\ell}\right]$ and $\left[L_{u}\right]$. As briefly discussed in Section 2.1.4, $\left[L_{\ell}\right]$ (resp. $\left[L_{u}\right]$ ) can not be defined as the infimum (resp. supremum) on the set of realizations $\left\{\left[L_{\sigma}^{\mathrm{app}}\left(\theta_{k}\right)\right]\right\}_{k=1}^{N_{s i m}}$ (resp. $\left.\left\{\left[L_{\epsilon}^{\mathrm{app}}\left(\theta_{k}\right)\right]\right\}_{k=1}^{N_{\text {sim }}}\right)$. Consequently, we propose to define these bounds as follows:

$$
\begin{aligned}
& {\left[L_{\ell}\right]=\underset{[L] \in \mathcal{C}_{a d}^{\ell}}{\arg \min } \sum_{k=1}^{N_{s i m}}\left\|\left[L_{\sigma}^{\mathrm{app}}\left(\theta_{k}\right)\right]-[L]\right\|_{\mathrm{F}},} \\
& {\left[L_{u}\right]=\underset{[L] \in \mathcal{C}_{a d}^{u}}{\arg \min } \sum_{k=1}^{N_{s i m}}\left\|[L]-\left[L_{\epsilon}^{\mathrm{app}}\left(\theta_{k}\right)\right]\right\|_{\mathrm{F}},}
\end{aligned}
$$

in which

$$
\begin{aligned}
& \mathcal{C}_{a d}^{\ell}=\left\{[L] \in \mathbb{M}_{n}^{+}(\mathbb{R}) \mid[L]<\left[L_{\sigma}^{\mathrm{app}}\left(\theta_{k}\right)\right], k=1, \ldots, N_{\text {sim }}\right\}, \\
& \mathcal{C}_{a d}^{u}=\left\{[L] \in \mathbb{M}_{n}^{+}(\mathbb{R}) \mid\left[L_{\epsilon}^{\mathrm{app}}\left(\theta_{k}\right)\right]<[L], k=1, \ldots, N_{\text {sim }}\right\} .
\end{aligned}
$$


Once the deterministic bounds $\left[L_{\ell}\right]$ and $\left[L_{u}\right]$ have been computed solving the optimization problems $(65-66)$, the realization $\left[L_{m}^{\mathrm{app}}\right]$ of the apparent tensor $\left[\mathbf{L}_{m}^{\text {app }}\right]$, associated with the considered realization of the microstructure, is finally defined as:

$$
\left[L_{m}^{\mathrm{app}}\right]=\underset{\left[L_{\ell}\right]<[L]<\left[L_{u}\right]}{\arg \min }\left\|<\sigma>_{M B C}-[L]<\epsilon>_{M B C}\right\| .
$$

\subsubsection{Identification of the probabilistic model}

Let $\Omega$ be a $0.3 \times 0.3$ square domain and let $N_{\text {sim }}=100$. The sets of realizations $\left\{\left[L_{\sigma}^{\mathrm{app}}\left(\theta_{k}\right)\right]\right\}_{k=1}^{100}$ and $\left\{\left[L_{\epsilon}^{\mathrm{app}}\left(\theta_{k}\right)\right]\right\}_{k=1}^{100}$ are computed using the methodology detailed in Section 4.3.1. Solving Eqs. (65-66) first yields estimates of the bounds (in megapascals):

$$
\begin{gathered}
{\left[\widetilde{L}_{\ell}\right]=10^{5}\left[\begin{array}{ccc}
1.0869 & 0.6068 & 0.0007 \\
0.6068 & 1.0884 & 0.0007 \\
0.0007 & 0.0007 & 0.2347
\end{array}\right],} \\
{\left[\widetilde{L}_{u}\right]=10^{5}\left[\begin{array}{ccc}
1.1487 & 0.5787 & 0.0013 \\
0.5787 & 1.1477 & -0.0003 \\
0.0013 & -0.0003 & 0.2825
\end{array}\right] .}
\end{gathered}
$$

For all the realizations of the volume averaged stress and strain vectors, solving Eq. (69), taking into account Eqs. (70-71) and Eq. (34), allows the corresponding realizations $\left[N\left(\theta_{1}\right)\right], \ldots,\left[N\left(\theta_{N_{\text {sim }}}\right)\right]$ of $[\mathbf{N}]$ to be computed. Consequently, estimates of parameters $[\underline{N}]$ and $\delta_{[\mathbf{K}]}$ of the probabilistic model can be readily determined using classical statistical estimates:

$$
\begin{gathered}
{[\underline{\tilde{N}}]=\frac{1}{N_{\text {sim }}} \sum_{k=1}^{N_{\text {sim }}}\left[N\left(\theta_{k}\right)\right],} \\
\widetilde{\delta}_{[\mathbf{K}]}=\widetilde{\delta}_{[\mathbf{N}]} \sqrt{n+1}\left\{1+\frac{(\operatorname{tr}[\underline{\tilde{N}}])^{2}}{\operatorname{tr}\left([\underline{\tilde{N}}]^{2}\right)}\right\}^{-1 / 2},
\end{gathered}
$$

in which

$$
\widetilde{\delta}_{[\mathbf{N}]}=\left\{\frac{1}{N_{s i m}\|[\underline{\tilde{N}}]\|_{\mathrm{F}}^{2}} \sum_{k=1}^{N_{s i m}}\left\|\left[N\left(\theta_{k}\right)\right]-[\underline{\tilde{N}}]\right\|_{\mathrm{F}}^{2}\right\}^{1 / 2} .
$$

It is found that:

$$
\widetilde{\delta}_{[\mathbf{N}]}=0.66,
$$


and

$$
[\underline{\tilde{N}}]=10^{-3}\left[\begin{array}{ccc}
0.2767 & 0.0879 & -0.0189 \\
0.0879 & 0.2214 & 0.0277 \\
-0.0189 & 0.0277 & 0.2366
\end{array}\right] .
$$

Making use of these parameters, numerical Monte-Carlo simulations of random matrix $[\mathbf{N}]$ can be readily performed and allow realizations of $[\mathbf{L}]$ to be obtained. Since the construction of the probabilistic model has been addressed for $[\mathbf{N}]$, it is worth comparing the sets of experimental and simulated realizations of the random apparent elasticity matrix $[\mathbf{L}]$. Let $\left[\widetilde{L}^{\text {exp }}\right]$ and $\left[\widetilde{L}^{\text {sim }}\right]$ be the statistical estimates of mean value $[\underline{L}]$ computed using the experimental and the simulated realizations respectively. It is found that:

$$
\left[\underline{\widetilde{L}}^{\text {exp }}\right]=10^{5}\left[\begin{array}{ccc}
1.1176 & 0.5907 & 0.0028 \\
0.5907 & 1.1230 & -0.0019 \\
0.0028 & -0.0019 & 0.2584
\end{array}\right]
$$

and

$$
\left[\underline{\widetilde{L}}^{s i m}\right]=10^{5}\left[\begin{array}{ccc}
1.1153 & 0.5944 & 0.0025 \\
0.5944 & 1.1194 & -0.0016 \\
0.0025 & -0.0016 & 0.2601
\end{array}\right] .
$$

It is seen that the mean model computed using the the probabilistic model reasonably matches the one estimated from the experimental realizations of $[\mathbf{L}]$. Similarly, the corresponding estimates $\widetilde{\delta}_{[\mathbf{L}]}^{\text {exp }}$ and $\widetilde{\delta}_{[\mathbf{L}]}^{\text {sim }}$ of $\delta_{[\mathbf{L}]}$ are given by:

$$
\widetilde{\delta}_{[\mathbf{L}]}^{e x p}=0.0108,
$$

and

$$
\widetilde{\delta}_{[\mathbf{L}]}^{s i m}=0.0093 .
$$

The relative error $\left|\widetilde{\delta}_{[\mathbf{L}]}^{\text {exp }}-\widetilde{\delta}_{[\mathbf{L}]}^{\text {sim }}\right| / \widetilde{\delta}_{[\mathbf{L}]}^{\text {exp }}$ is equal to 0.1389 , showing a good agreement between the model predictions and the experimental data as well. Furthermore, it is seen that the level of statistical fluctuations exhibited by the random elasticity tensor is small. This fact can be explained by noticing that (i) the RVE associated with polycrystalline microstructures is generally small (which is here confirmed by the closeness of the upper and lower bounds), and that (ii) the Aluminium single crystal presents a cubic material symmetry (and is relatively close to isotropy, since the Zener anisotropy index [40], defined as $2\left[L^{\mathrm{Al}}\right]_{44} /\left(\left[L^{\mathrm{Al}}\right]_{11}-\left[L^{\mathrm{Al}}\right]_{12}\right)$, is equal to 1.2$)$, so that the crystallographic orientation does not induce large fluctuations in the elasticity tensors of the grains. Indeed, further numerical experiments show that considering a single crystal whose Zener anisotropy index is much 
greater than 1 yields a larger level of statistical fluctuations of the apparent matrices (in the case of a copper crystal, for which the Zener anisotropy index is equal to 3.2 , one has $\widetilde{\delta}_{[\mathbf{L}]}^{\text {exp }} \approx 0.09$ for instance), all other parameters (e.g. the probability distribution of the Euler angles) being fixed. This observation is consistent with the results provided in [23].

\subsection{Application to a beam with mesoscopic randomness}

For illustration purposes, we consider the open bounded domain $\mathcal{D}$ of $\mathbb{R}^{2}$ such that $\mathcal{D}=] 0,20[\times] 0,1[$ (in millimeters) and then, $n=3$. Null displacement Dirichlet boundary conditions are applied to the boundary $\Gamma_{D}$ defined as $\Gamma_{D}=\left\{\mathbf{x} \in \mathcal{D} \mid x_{1}=0\right\}$, while a static load point $(0,-0.01)$ (in newtons) is prescribed at the point of coordinates $(20,1)$.

The elasticity tensor random field is defined with reference to the polycrystalline microstructure characterized in Section 4.1 (and simulated as discussed in Section 4.2). Consequently, following the identification carried out in Section 4.3, the mean function is such that $[\underline{\mathbf{N}}(\mathbf{x})]=[\underline{\tilde{N}}]$ (see Eq. (76)), the dispersion parameter $\delta_{[\mathbf{N}]}(\mathbf{x})$ is given by $\delta_{[\mathbf{N}]}(\mathbf{x})=\delta_{[\mathbf{N}]}$ (see Eq. (75)) and the deterministic fields $\mathbf{x} \mapsto\left[L_{\ell}(\mathbf{x})\right]$ and $\mathbf{x} \mapsto\left[L_{u}(\mathbf{x})\right]$ are such that $\left[L_{\ell}(\mathbf{x})\right]=\left[\widetilde{L}_{\ell}\right]$ and $\left[L_{u}(\mathbf{x})\right]=\left[\widetilde{L}_{u}\right]$ (see Eqs. (70)-(71)) for all $\mathbf{x}$ in $\mathcal{D}$. The spatial correlation lengths are assumed to be such that $l_{i j}^{k}=\ell_{c}=1$ (millimeter) for all $i, j$ and $k$. Domain $\mathcal{D}$ is discretized using $40 \times 2=80$ 4-nodes isoparametric finite elements (with 4 integrating points), as shown in Fig. 6.

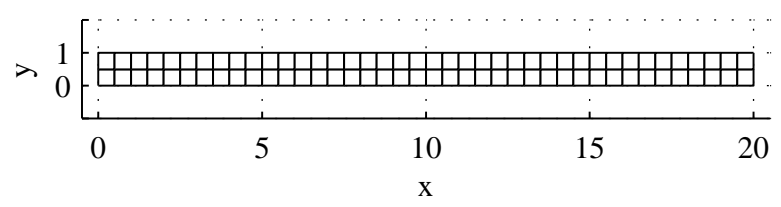

Figure 6: Plot of the mesh of domain $\mathcal{D}$.

The $\mathbb{R}^{2}$-valued displacement random field $\mathbf{x} \mapsto \mathbf{u}(\mathbf{x})=\left(u_{1}(\mathbf{x}), u_{2}(\mathbf{x})\right)$ is characterized solving the stochastic boundary value problem with $n_{m c}$ Monte-Carlo simulations (see Section 3.2). In order to illustrate the effect of the boundedness constraints, the nonparametric approach derived in [34] is also considered and is parameterized by Eqs. (77-79) (assuming the same correlation structure as for the proposed approach). 
Let $\mathcal{X}$ be the random vector of the $u_{2}$-displacement of the nodes located in the central line of the beam. The convergence of the statistical estimate of $\sqrt{\mathrm{E}\left\{\|\mathcal{X}\|_{E}^{2}\right\}}$ (in which $\|\cdot\|_{E}$ is the usual Euclidean norm) with respect to $n_{m c}$ is first studied and shown in Fig. 7. It is seen that a reasonable level of convergence is reached for $n_{m c}=3000$ simulations.

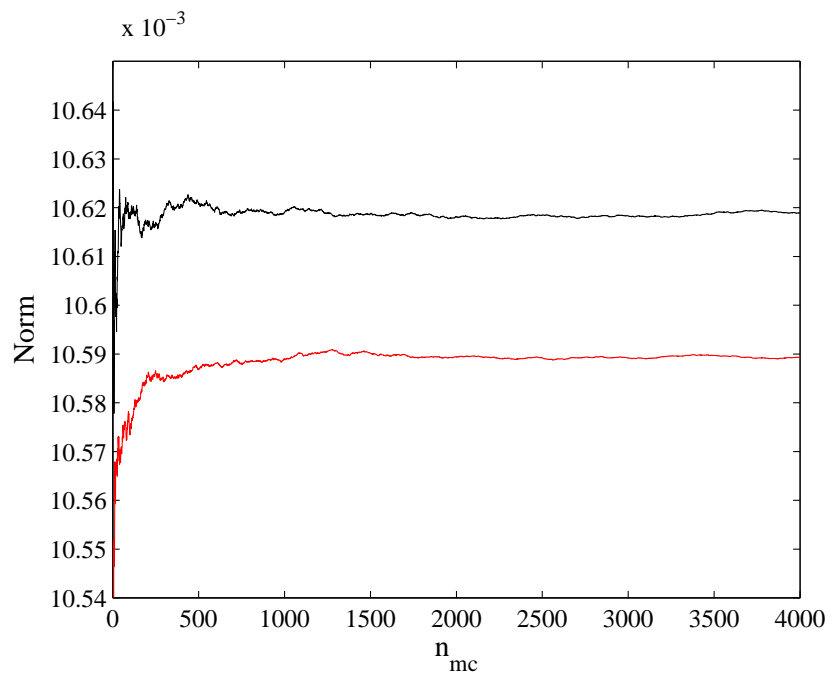

Figure 7: Convergence of the statistical estimate of $\sqrt{\mathrm{E}\left\{\|\mathcal{X}\|_{E}^{2}\right\}}$ with respect to the number of Monte-Carlo simulations: proposed approach with boundedness contraints (black line), Soize's nonparametric approach (red line).

Clearly, for a given level of statistical fluctuations, the effect of the boundedness constraints is more significant for small levels of probability. This point is illustrated in Figs. 8 and 9, where the confidence region (associated with probability level $P=0.99$ ) of the transverse displacement of the central line and the probability density functions of the maximum transverse displacement are respectively plotted for the proposed approach (black line) and Soize's nonparametric model (dashed line). Obviously, the observed discrepancy between the two probabilistic models is all the more small that the level of statistical fluctuations of the elasticity tensor random field is small (see Eq. (79)).

\section{Conclusion}

In this paper, we have addressed the construction of a prior stochastic model for non-Gaussian deterministically-bounded positive-definite matrix- 


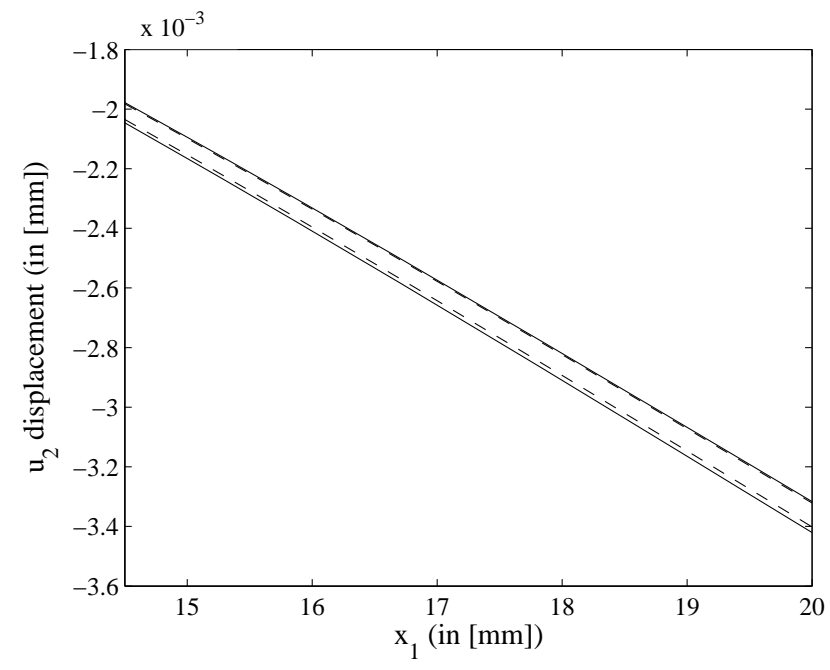

Figure 8: Confidence region (associated with probability level $P=0.99$ ) of the transverse displacement of the central line for the proposed approach (black line) and Soize's nonparametric model (dashed line).

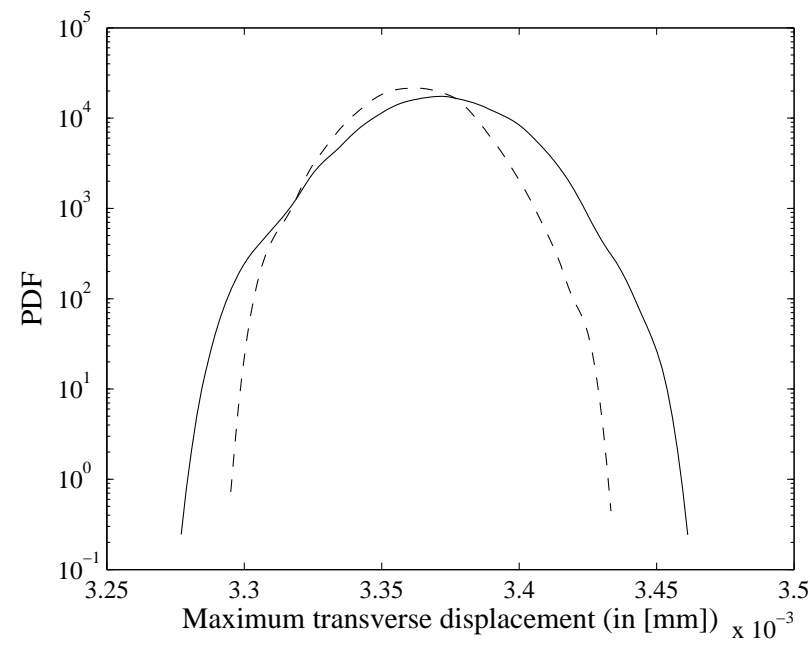

Figure 9: Semilog scale plot of the probability density functions of the maximum transverse displacement $\max _{\mathbf{x} \in \mathcal{D}}\left|u_{2}(\mathbf{x})\right|$ (in millimeters) for the proposed approach (black line) and Soize's nonparametric model (dashed line). 
valued random fields in the context of mesoscale modeling of heterogeneous elastic microstructures. With reference to Huet's Partition Theorem, we first discussed the definition of bounds that are used for specifying the support of the probability density function for the mesoscopic random elasticity tensor. Emphasis is put on the random or deterministic nature of the bounds, which has an important consequence for the construction of the probabilistic model. In particular, a numerical procedure, allowing one to define approximate deterministic bounds in the general case of a stochastic boundedness constraint, is proposed. Considering next the case of deterministic lower and upper bounds, two random matrix models are presented. The first one, previously derived in the literature, specifically allows prescribing the behavior of the distribution around the bounds. Making use of an alternative formulation of the boundedness constraints, a second probabilistic model is proposed and allows extending the modeling to deterministically-bounded positive-definite matrix-valued random fields. Such an extension is readily performed making use of the class of random fields introduced in [34]. It is worth mentioning that while the probabilistic model was derived for modeling mesoscale random elasticity tensors, it is also suitable for other applications involving non-Gaussian positive-definite matrix-valued random fields, for which similar boundedness constraints hold (such as random permeability tensors; see [4]). The overall methodology is finally exemplified through the calibration of the prior probabilistic model, performed using an experimental database obtained from EBSD measurements on Al alloy. In accordance with the literature, it is shown that the level of statistical fluctuations of the apparent tensor is small because of the material symmetry exhibited by the aluminium crystal (which is relatively close to isotropy). A simple numerical application, carried out considering both the proposed probabilistic model and the class of random fields defined in [34], is also provided.

The procedure leading up to the foregoing prior probabilistic model provides a compact stochastic representation for a non-Gaussian tensor-valued random field. Uncertainty propagation procedures can be used to explore the dependence of model-based predictions on this prior stochastic model. It is important to note that the smallest statistical fluctuations at finer (microscopic) scales are smoothed at the mesoscale considered in the calibration procedure and consequently, cannot be accounted for and consequently, cannot be propagated. The proposed stochastic model at mesoscale is, therefore, well adapted for taking into account physical phenomena occurring at this scale and characterizing the consequences induced at the macroscale. 


\section{Acknowledgments}

This work was supported by a MURI administered by AFOSR on Health Monitoring and Materials Damage Prognosis for Metallic Aerospace Propulsion and Structural Systems, and by the French Research Agency (grant number: ANR-2010-BLAN-0904).

\section{References}

[1] T. W. Anderson. Introduction to multivariate statistical analysis. Wiley, New-York, 1958.

[2] S. Das and R. Ghanem. A bounded random matrix approach for stochastic upscaling. Multiscale Modeling $\&$ Simulation, 8(1):296-325, 2009.

[3] J. Dattorro. Convex Optimization \& Euclidean Distance Geometry. Meboo Publishing USA, Palo Alto, CA, 2005.

[4] X. Du and M. Ostoja-Starzewski. On the size of representative volume element for darcy law in random media. Proc. R. Soc. A, 462:29492963, 2006.

[5] R. Ghanem and P. Spanos. Stochastic finite elements: a Spectral Approach. Springer, New-York, 1991.

[6] J. Guilleminot and C. Soize. A stochastic model for elasticity tensors with uncertain material symmetries. International Journal of Solids and Structures, 47(22-23):3121-3131, 2010.

[7] A. K. Gupta and D. K. Nagar. Matrix variate distributions. Chapman and Hall-CRC, 2000.

[8] S. Hazanov and C. Huet. Order relationships for boundary conditions effect in heterogeneous bodies smaller than the representative volume. Journal of the Mechanics and Physics of Solids, 42(12):1995-2011, 1994.

[9] R. Hill. Elastic properties of reinforced solids: some theoretical principles. Journal of the Mechanics and Physics of Solids, 11:357-372, 1963. 
[10] C. Huet. Application of variational concepts to size effects in elastic heterogeneous bodies. Journal of the Mechanics and Physics of Solids, 38(6):813-841, 1990.

[11] R. L. Iman and W. Conover. A distribution-free approach to inducing rank correlation among input variables. Communications in Statistics - Simulation and Computation, 11:311-334, 1982.

[12] M. Jardak and R. G. Ghanem. Spectral stochastic homogenization of divergence-type pdes. Computer Methods in Applied Mechanics and Engineering, 193(6-8):429-447, 2004.

[13] E. T. Jaynes. Information theory and statistical mechanics. Physical Review, 106(4):620-630, 1957.

[14] E. T. Jaynes. Information theory and statistical mechanics. Physical Review, 108(2):171-190, 1957.

[15] J. Löfberg. Yalmip: A toolbox for modeling and optimization in MATLAB. In Proceedings of the IEEE Conference on Computer Aided Control Systems Design (CACSD), Taipei, Taiwan, 2004.

[16] T. Mura. Micromechanics of defects in solids. M. Nijhoff Publ., The Hague, The Netherlands, 1987.

[17] D. K. Nagar and A. K. Gupta. Matrix-variate kummer-beta distribution. Journal of the Australian Mathematical Society, 73:11-25, 2002.

[18] R. M. Neal. Slice sampling. The annals of Statistics, 31(3):705-767, 2003.

[19] S. Nemat-Nasser and M. Hori. Micromechanics: overall properties of heterogeneous materials. North-Holland, 1993.

[20] A. Okabe. Spatial tessellations : concepts and applications of Voronoi diagram. Wiley series in probability and statistics. Wiley, 2000.

[21] M. Ostoja-Starzewski. Microstructural Randomness and Scaling in Mechanics of Materials. Chapman and Hall-CRC, 2008.

[22] M. Parra Garcia, C. Luo, A. Noshadravan, A. Keck, R. Teale, A. Chattopadhyay, and P. Peralta. Microstructure representation and material characterization for multiscale finite element simulations of local mechanical behavior in damaged metallic structures. In Proceedings of 
SPIE, the International Society for Optical Engineering, San Diego, California, USA, 10-12 March 2008.

[23] S. I. Ranganathan and M. Ostoja-Starzewski. Scaling function, anisotropy and the size of rve in elastic random polycrystals. Journal of the Mechanics and Physics of Solids, 56:2773-2791, 2008.

[24] K. Sab. On the homogenization and the simulation of random materials. European journal of mechanics A/Solids, 11(5):585-607, 1992.

[25] S. Sankaran and N. Zabaras. A maximum entropy approach for property prediction of random microstructures. Acta Materialia, 54:2265-2276, 2006 .

[26] T. H. Scheike. Anisotropic growth of voronoi cells. Advances in applied probability, 26(1):43-53, 1994.

[27] A. J. Schwartz. Electron backscatter diffraction in materials science. Kluwer Academic, 2000.

[28] R.J. Serfling. Approximation Theorems of Mathematical Statistics. John Wiley \& Sons, 1980.

[29] C. E. Shannon. A mathematical theory of communication. Bell System Technical Journal, 27:379-423/623-659, 1948.

[30] M. Shinozuka. Simulations of multivariate and multidimensional random processes. Journal of the Acoutical Society of America, 39(1):357367, 1971.

[31] C. Soize. A nonparametric model of random uncertainties on reduced matrix model in structural dynamics. Probabilistic Engineering $\mathrm{Me}$ chanics, 15(3):277-294, 2000.

[32] C. Soize. Maximum entropy approach for modeling random uncertainties in transient elastodynamics. Journal of the Acoutical Society of America, 109(5):1979-1996, 2001.

[33] C. Soize. Non-gaussian positive-definite matrix-valued random fields for elliptic stochastic partial differential operators. Computer Methods in Applied Mechanics and Engineering, 195:26-64, 2006. 
[34] C. Soize. Tensor-valued random fields for meso-scale stochastic model of anisotropic elastic microstructure and probabilistic analysis of representative volume element size. Probabilistic Engineering Mechanics, $23: 307-323,2008$.

[35] C. Soize. Generalized probabilistic approach of uncertainties in computational dynamics using random matrices and polynomial chaos decompositions. International Journal of Numerical Methods in Engineering, 81(8):939-970, 2010.

[36] Q.A. Ta, D. Clouteau, and R. Cottereau. Modeling of random anisotropic elastic media and impact on wave propagation. European Journal of Computational Mechanics, 19(1-2-3):241-253, 2010.

[37] M. Tootkaboni and L. Graham-Brady. A multi-scale spectral stochastic method for homogenization of multi-phase periodic composites with random material properties. International Journal of Numerical Methods in Engineering, 83:59-90, 2010.

[38] S. Torquato. Random Heterogeneous Materials: Microstructure and Macroscopic Properties. Springer, New-York, 2002.

[39] L. Vandenberghe and S. Boyd. Semidefinite programming. SIAM Review, 38(1):49-95, 1996.

[40] C. Zener. Elasticity and Anelasticity of Metals. University of Chicago Press, Chicago, 1948. 